\title{
Critical Analysis of Particle Detection Artifacts in Synaptosome Flow Cytometry
}

\author{
(1)Benjamin D. Hobson ${ }^{1,2,3}$ and Peter A. Sims ${ }^{1,2,4}$
}

https://doi.org/10.1523/ENEURO.0009-19.2019

${ }^{1}$ Department of Systems Biology, Columbia University Irving Medical Center, New York, NY 10032, ${ }^{2}$ Department of Biochemistry and Molecular Biophysics, Columbia University Irving Medical Center, New York, NY $10032,{ }^{3}$ Medical Scientist Training Program, Columbia University Irving Medical Center, New York, NY 10032, and ${ }^{4}$ Sulzberger Columbia Genome Center, Columbia University Irving Medical Center, New York, NY 10032

\begin{abstract}
Flow cytometry and fluorescence-activated sorting are powerful techniques that hold great promise for studying heterogeneous populations of submicron particles such as synaptosomes, but many technical challenges arise in these experiments. To date, most flow cytometry studies of synaptosomes have relied on particle detection using forward scatter (FSC) measurements and size estimation with polystyrene (PS) bead standards. However, these practices have serious limitations, and special care must be taken to overcome the poor sensitivity of conventional flow cytometers in the analysis of submicron particles. Technical artifacts can confound these experiments, especially the detection of multiple particles as a single event. Here, we compared analysis of P2 crude synaptosomal preparations from murine forebrain on multiple flow cytometers using both FSC-triggered and fluorescence-triggered detection. We implemented multicolor fluorescent dye-based assays to quantify coincident particle detection and aggregation, and we assessed the false colocalization of antigens in immunostaining analyses. Our results demonstrate that fluorescence triggering and proper dilution can control for coincident particle detection, but not particle aggregation. We confirmed previous studies showing that FSCbased size estimation with PS beads underestimates biological particle size, and we identified pervasive aggregation in the FSC range analyzed in most synaptosome flow cytometry studies. We found that analyzing P2 samples in sucrose/EDTA/tris (SET) buffer reduces aggregation compared to PBS, but does not completely eliminate the presence of aggregates, especially in immunostaining experiments. Our study highlights challenges and pitfalls in synaptosome flow cytometry and provides a methodological framework for future studies.
\end{abstract}

Key words: flow cytometry; synaptosomes

\section{Significance Statement}

Synaptosomes are an invaluable model for synaptic biology, but these synaptic particles are traditionally analyzed in bulk preparations rather than at the level of single particles. Although flow cytometry is a powerful technique for high-throughput particle analysis, submicron particles present unique challenges. Here, the authors investigate key elements of synaptosome flow cytometry experiments, especially those related to artifacts that confound the analysis of single synaptosomes. They identify aggregation as especially problematic and implement methods to minimize its impact on flow cytometry analysis. 


\section{Introduction}

Synaptosomes are synaptic particles consisting of resealed presynaptic nerve terminals that often remain bound to postsynaptic elements (Whittaker, 1993). These structures were originally isolated by homogenization of brain tissue in isotonic sucrose (Gray and Whittaker, 1962), and have been further purified using a variety of filtration and density gradient centrifugation procedures (Hollingsworth et al., 1985; Dunkley et al., 1986). Synaptosomes retain functional properties such as membrane potential and depolarization-induced neurotransmitter release, making them key model systems for fundamental synaptic biology (Whittaker, 1993). However, studies of synaptosomes in bulk are limited by the purity of the preparations, which contain a mixture of synapse types (e.g., glutamatergic, GABAergic, etc.) as well as contaminating neuronal and glial membranes. In principle, high-throughput purification and analysis of single synaptosomes is a powerful tool for addressing the incredible heterogeneity of the billions of synapses in mammalian brains.

Flow cytometry employs a pressurized fluidic system to pass suspensions of cells or particles through an optical flow cell, where scattered light and fluorescence measurements are collected for each particle. Analysis of submicron particles on conventional flow cytometers faces a variety of limitations and pitfalls due to the limited sensitivity of these instruments (Nolan, 2015). Event detection in flow cytometers, known as triggering, occurs when a particle passing through the flow cell causes the trigger parameter to rise above a manually set threshold. The trigger parameter, usually forward scatter (FSC), must have a minimum threshold set as to avoid electronic and buffer noise, but this limits detection of submicron particles. It is generally accepted that conventional flow cytometers have a lower limit of FSC-triggered detection around 300- to 500-nm PS beads (van der Pol et al., 2013), but this does not correspond to the size of biological particles that can be detected (Nolan, 2015; Lannigan et al., 2016). FSC intensity for submicron particles depends on many factors besides particle size, especially

Received January 7, 2019; accepted April 27, 2019; First published May 22, 2019

The authors declare no competing financial interests.

Author contributions: B.D.H. and P.A.S. designed research; B.D.H. performed research; B.D.H. analyzed data; B.D.H. and P.A.S. wrote the paper.

This work was supported by the National Institute of General Medical Sciences Grant T32GM007367 (to B.D.H.), the National Institute on Drug Abuse Grant 1F30DA047775-01 (to B.D.H.), and the National Institute of Neurological Disorders and Stroke Grant R01NS103473 (to P.A.S.). Research reported in this publication was performed in the CCTI Flow Cytometry Core, supported in part by the Office of the Director, National Institutes of Health under Awards S10OD020056. These studies used the resources of the Cancer Center Flow Core Facility funded in part through Center Grant P30CA013696.

Acknowledgements: We thank Siu-Hong Ho, Lu Caisheng, and Wei Wang for excellent technical support in the CCTI Flow Cytometry Core.

Correspondence should be addressed to Benjamin D. Hobson at bh2573@columbia.edu or Peter A. Sims at pas2182@columbia.edu

https://doi.org/10.1523/ENEURO.0009-19.2019

Copyright @ 2019 Hobson and Sims

This is an open-access article distributed under the terms of the Creative Commons Attribution 4.0 International license, which permits unrestricted use, distribution and reproduction in any medium provided that the original work is properly attributed. refractive index, which is generally $1.59-1.61$ for PS beads, 1.40-1.46 for silica beads, and ranges from 1.33 to 1.40 for cell-derived particles (Nolan, 2015). Biological particles therefore scatter light $\sim 10$-fold less efficiently than PS beads (Chandler et al., 2011), and direct comparison of PS bead FSC intensity has been shown to underestimate the size of biological particles (Chandler et al., 2011; van der Pol et al., 2012; Simonsen, 2016).

In addition to difficulties with size estimation using light scatter, numerous reports have identified particle detection artifacts in submicron flow cytometry. Particles below the trigger threshold can be detected when analyzed at high concentrations, a phenomenon known as coincidence or "swarm detection" (van der Pol et al., 2012; Nolan and Stoner, 2013). While coincidence is caused by the simultaneous presence of multiple, single particles in the path of the laser, others have reported detection of single events comprised of multiple, aggregated submicron particles (Erdbrügger et al., 2014). Although these artifacts have been recognized in the cell-derived microparticle community (Nolan, 2015; Lannigan et al., 2016), most flow cytometry studies of synaptosomes have not addressed triggering or detection artifacts (Gylys et al., 2000; Fein et al., 2008; Postupna et al., 2014; Prieto et al., 2017). Despite claims that single synaptosomes can be detected by FSC triggering and identified apart from contaminating particles based solely on FSC signal (Gylys et al., 2004; Gylys and Bilousova, 2017), this has been a point of recent controversy (see comments on Prieto et al., 2017). In contrast to these studies, Biesemann and Herzog found that sorting of FSC-triggered events bearing genetically encoded vesicular glutamate transporter 1 (VGLUT1) fluorescence yielded samples contaminated with GABAergic synaptosomes and myelin (Biesemann, 2010; his Results 3.4, Fig. 14, pp 86-87). Instead, fluorescence triggering and sorting events below the FSC trigger threshold yielded a VGLUT1 synaptosomal sample of unprecedented purity (Biesemann et al., 2014). The flow cytometry protocols published by these groups are clearly at odds in terms of the best practices to ensure detection, analysis, and sorting of single synaptosomes (Gylys and Bilousova, 2017; Luquet et al., 2017).

Here, we present a critical flow cytometry analysis of P2 crude synaptosome preparations on two cytometers, the BD Influx and BD LSRFortessa. We investigated several experimental aspects of synaptosome flow cytometry, including: (1) FSC-triggered and fluorescence-triggered particle detection, (2) FSC-based size estimation with PS and silica bead standards, (3) range of particle concentration, (4) coincidence and aggregation as causes of false double-positive events, (5) sucrose and PBS buffers for sample preparation and acquisition, and (6) false colocalization of antigens in immunostained samples. Our study highlights technical challenges and identifies methods to minimize their impact on experimental results.

\section{Materials and Methods}

\section{Animals}

Male C57BL/6J mice (6-10 weeks old) were used in all experiments. Mice were housed on a 12/12 h light/dark 
Table 1. Optical configuration of the LSRFortessa

\begin{tabular}{|c|c|c|c|c|}
\hline \multicolumn{5}{|c|}{ LSRFortessa optics } \\
\hline Laser & Band pass filter & Dichroic filter & Detector name & Fluorophores used in this study \\
\hline $405 \mathrm{~nm}$ & $450 / 50$ & Blank & Pacific Blue & Violet amine-reactive dye \\
\hline $488 \mathrm{~nm}$ & $488 / 10$ & Blank & SSC & \\
\hline $488 \mathrm{~nm}$ & $530 / 30$ & 505LP & FITC & Alexa Fluor 488, Calcein AM \\
\hline $488 \mathrm{~nm}$ & $710 / 50$ & 685LP & PerCP-Cy5.5 & FM4-64 \\
\hline $561 \mathrm{~nm}$ & $582 / 12$ & Blank & $\mathrm{PE}$ & Alexa Fluor 555, Calcein red \\
\hline $633 \mathrm{~nm}$ & $670 / 30$ & Blank & APC & Alexa Fluor 647, MitoTracker Deep Red FM \\
\hline
\end{tabular}

cycle with food and water available ad libitum. All animal procedures were performed in accordance with the Columbia University Institutional Animal Care and Use Committee and followed National Institutes of Health guidelines.

\section{P1 crude nuclei and P2 crude synaptosome preparations}

Preparation of the P2 crude synaptosome fraction was performed using standard procedures (Gray and Whittaker, 1962). Mice were sacrificed by cervical dislocation, after which forebrains were rapidly dissected and placed in 10 volumes of ice-cold buffer consisting of $0.32 \mathrm{M}$ sucrose, $4 \mathrm{mM}$ HEPES ( $\mathrm{pH}$ 7.4), and protease inhibitors (cOmplete EDTA-free protease inhibitor, Roche). Tissue was homogenized on ice in a glass-glass dounce homogenizer with 10 gentle strokes of loose and tight clearance pestles. All subsequent purification steps were performed on ice or at $4^{\circ} \mathrm{C}$ unless otherwise specified. The homogenate was centrifuged at $1000 \times g$ (Eppendorf 5424R) for $10 \mathrm{~min}$ to remove nuclei and cellular debris, yielding a P1 pellet and an S1 supernatant.

A crude nuclei preparation was prepared for flow cytometry according to established procedures (Krishnaswami et al., 2016). The P1 pellet was resuspended in ice cold buffer containing $250 \mathrm{mM}$ sucrose, $25 \mathrm{mM} \mathrm{KCl,} 5$ $\mathrm{mM} \mathrm{MgCl}, 10 \mathrm{mM}$ Tris (pH 7.4), $1 \mu \mathrm{M}$ DTT, 0.1\% Triton $\mathrm{X}-100$, and $1 \mu \mathrm{M}$ Hoechst 33342 and homogenized again with 10 strokes of the tight pestle to facilitate release of nuclei. The homogenate was rotated for $15 \mathrm{~min}$ at $4^{\circ} \mathrm{C}$, filtered through a 40- $\mu \mathrm{m}$ cell strainer cap, and centrifuged at $500 \times g$ (Eppendorf 5424R) for $5 \mathrm{~min}$ to yield a crude nuclear pellet.

The S1 supernatant was further centrifuged at $10,000 \times$ $g$ (Eppendorf 5424R) for 20 min to obtain the crude synaptosome pellet (P2). P2 pellets were cryopreserved by resuspension in $4 \mathrm{mM}$ HEPES/0.32 $\mathrm{M}$ sucrose buffer + $5 \%$ DMSO and slowly frozen to $-80^{\circ} \mathrm{C}$ using an isopropanol freezing container. Frozen synaptosomes were used within two months. This protocol, when combined with rapid thawing at $37^{\circ} \mathrm{C}$ on the day of the experiment, has been shown to preserve synaptosome function and morphology (Gleitz et al., 1993; Daniel et al., 2012). After thawing, all experiments were conducted with either PBS (137 mM NaCl, $2.7 \mathrm{mM} \mathrm{KCl,} 8 \mathrm{mM} \mathrm{Na}_{2} \mathrm{HPO}_{4}$, and $2 \mathrm{mM}$ $\mathrm{KH}_{2} \mathrm{PO}_{4}$ ) or sucrose/EDTA/tris (SET) buffer (320 mM sucrose, $5 \mathrm{mM}$ Tris, and $1 \mathrm{mM}$ EDTA).

\section{Flow cytometry instrumentation and setup}

All flow cytometry data acquisition was conducted using the instrument software FACSDiva (BD Biosciences). All flow cytometry data analysis including gating, quantification, and generation of density plots/histograms was performed using FCS Express 6 (De Novo Software). Number of events, \% of all events, and channel statistics (median, geometric mean, SD, etc.) for all gates were exported using "Batch Export" for further statistical analysis.

All data acquisition on LSRFortessa (hereafter Fortessa, BD Biosciences) was conducted using the lowest possible sample pressure settings. Optical configuration employed and fluorophores detected in these channels are summarized in Table 1.

The powers of the lasers in the Fortessa are $405 \mathrm{~nm} 100$ mW, 488 nm 50 mW, 561 nm 100 mW, 640 nm 100 mW. Detector voltages underwent minor fluctuations over the course of these studies to maintain comparable sample fluorescence and FSC/SSC values for the fluorescent microspheres, which were run at the beginning of each experiment. Approximate detector voltages were as follows: FSC (300), SSC (265), Pacific Blue (407), FITC (588), PerCP-Cy5.5 (500), PE (511), and APC (537).

The BD Influx (hereafter Influx, BD Biosciences) was operating using a $100-\mu \mathrm{m}$ nozzle at 11.1 psi. The sample pressure differential was kept as low as possible. The frequency was set at $25.8 \mathrm{kHz}$, piezo amplitude between 5 and 10. Optical configuration employed and fluorophores detected in these channels are summarized in Table 2.

The power of the lasers in the Influx are $405 \mathrm{~nm} 100$ mW, 488 nm 200 mW, 561 nm 120 mW, 640 nm 120 mW. Detector voltages underwent minor fluctuations over the course of these studies to maintain comparable sample fluorescence and FSC/SSC values for the bead standards. Approximate detector voltages were as follows: FSC (22.70), SSC (25.77), BV421 (52.55), FITC (44.51), PerCP-Cy5.5 (41.68), PE (63.65), APC (54.78).

In the annual preventive and maintenance conducted by the $B D$ engineer, a quality control $(\mathrm{QC})$ is run with the six-peak rainbow beads. In the daily setup, the QC setting is retrieved and the daily alignment is compared with the QC setting. The complete overlay of the six peaks with the QC setting indicates the proper alignment of the laser. Drop delay is done with the drop delay beads and the accudrop camera, a step which calculates the distance between the laser interrogation point and the charging point. 
Table 2. Optical configuration of the Influx

\begin{tabular}{|c|c|c|c|}
\hline \multicolumn{4}{|c|}{ Influx optics } \\
\hline Laser & Band pass filter & Detector name & Fluorophores used in this study \\
\hline $405 \mathrm{~nm}$ & $460 / 50$ & BV421 & Hoechst 33342 , Violet amine-reactive dye \\
\hline $488 \mathrm{~nm}$ & $488 / 10$ & SSC & \\
\hline $488 \mathrm{~nm}$ & $530 / 40$ & FITC & Alexa Fluor 488, Calcein AM \\
\hline $488 \mathrm{~nm}$ & $692 / 40$ & PerCP-Cy5.5 & FM4-64 \\
\hline $561 \mathrm{~nm}$ & $589 / 29$ & PE & Alexa Fluor 555, Calcein red \\
\hline $638 \mathrm{~nm}$ & $670 / 30$ & APC & Alexa Fluor 647, MitoTracker Deep Red FN \\
\hline
\end{tabular}

Daily setup also includes running a mixture of fluorescent PS bead standards (Bangs Labs, Inc.) and a mixture of non-fluorescent silica and fluorescent PS bead standards (Apogee Flow Systems). To ensure reproducibility in FSC-positioning of samples across experiments, the positions of bead populations on FSC versus SSC and FSC versus FITC (green fluorescence detector for PS beads) plots are maintained within tight gates stored in the QC workspace. Only occasionally did this require minor adjustments to detector voltages.

\section{Flow cytometry gating}

Gating of bead populations was performed using density plots of FSC versus SSC and FSC versus FITC (green fluorescence detector for PS beads). Gating of all fluorescent channels was performed using a polygon gate on a bivariate density plot of FSC versus fluorophore. Boolean logic gating was used to assess all intersections of positive versus negative for each fluorophore (e.g., doublenegative, calcein $\mathrm{AM}+/$ calcein red-, calcein $\mathrm{AM}$-/calcein red + , double-positive). Where applicable, hierarchical gating was used to assess all intersections of positive versus negative for each fluorophore within each FSC gate.

\section{FSC-triggered and FM4-64-triggered detection}

On both the Influx and Fortessa, the FSC voltages were set to place 500-nm PS beads just left of center on the FSC axis, near $10^{2}$ on the Influx and near $10^{3}$ on the Fortessa. This enabled the beads to be detected just above the FSC noise threshold while also allowing $800-\mathrm{nm}$ PS and 1300-nm Sil beads to be collected on scale. Critically, this also allowed for a significant amount of floor space on the FSC axis below the FSC noise threshold, which became accessible on fluorescence triggering with FM4-64.

For FSC triggering on the Influx and Fortessa, the FSC trigger threshold was first lowered to the minimum value on the instrument while running clean PBS to define the range of electronic, optical, buffer, and all other sources of FSC noise. After collecting $\sim 10,000$ events, the FSC voltage was raised to the minimum level just above the FSC noise threshold such that event rates were $<10$ events/s while running clean PBS. Using the configurations described above, the FSC-trigger threshold was generally $250-300$ on the Fortessa and generally $0.9-1.10$ on the Influx.

FM4-64 fluorescent triggering was implemented on the Influx and Fortessa using a procedure similar to FSC triggering. FM4-64 was obtained from ThermoFisher, re- constituted at $100 \mu \mathrm{g} / \mu \mathrm{l}$, and diluted into PBS or sucrose buffer at $1.5 \mu \mathrm{g} / \mathrm{ml}$. This concentration was previously shown to be effective for fluorescence triggering of synaptosomes (Biesemann et al., 2014; Luquet et al., 2017). We found optimal detection of FM4-64 fluorescence in the PerCP-Cy5.5 channel and used this channel for triggering on the Influx and Fortessa. As with FSC triggering, the fluorescence trigger threshold was first lowered to a minimum while running clean buffer + FM4-64 to define the noise range. The fluorescent trigger threshold was raised to the minimum level above the noise range such that event rates were $<10$ events/s while running buffer + FM4-64. Using the configurations described above, the FM-trigger threshold was generally $120-150$ on the Fortessa and generally $0.35-0.40$ on the Influx.

\section{Fluorescence-activated sorting}

For sorting on the Influx, populations of interest were gated using the FACSDiva software. For bead doublepositive and double calcein-positive sorting, the event rate was $<5000$ events/s. The sort was conducted using 1.0 Drop Pure mode. At least 20,000 events were sorted directly into FACS tubes, which were then used to reanalyze the sorted samples. Reanalysis was conducted $<15$ min after sorting using the exact same cytometer settings, and at least 1500 events were re-analyzed.

\section{Multicolor labeling and mixing assays}

Cryopreserved P2 samples were rapidly thawed at $37^{\circ} \mathrm{C}$, washed in PBS, and centrifuged at $10,000 \times g$ (Eppendorf 5424R) for $5 \mathrm{~min}$ at $4^{\circ} \mathrm{C}$. Pellets were resuspended in PBS and equally divided into separate tubes for single dye labeling. Synaptosomes were incubated at room temperature in PBS supplemented with one of the following dyes: calcein AM for $15-30$ min at a final concentration of $1 \mu \mathrm{M}$, calcein red-orange AM (hereafter calcein red) for 15-30 min at a final concentration of 1.9 $\mu \mathrm{M}$, violet amine-reactive dye for $30 \mathrm{~min}$ (1:1000 from $50-\mu$ l stock, as per manufacturer's guidelines), or MitoTracker Deep Red FM for 30 min at a final concentration of $500 \mathrm{nM}$. Synaptosomes were returned to ice and subsequently washed and re-centrifuged twice with ice-cold PBS to remove residual dye. All samples were maintained on ice and protected from light after labeling. After the second wash, pellets were resuspended in equal volumes of PBS + $1.5 \mu \mathrm{g} / \mathrm{ml} \mathrm{FM4-64} \mathrm{and} \mathrm{mixed} \mathrm{in} \mathrm{equal} \mathrm{volumes}$ to generate mixtures of single color-labeled synaptosomes. In most experiments, these mixtures were recentrifuged and resuspended before passing the suspension through a $40-\mu \mathrm{m}$ cell strainer cap before flow 
cytometry. In time lapse experiments, individual samples were passed through a $40-\mu \mathrm{m}$ cell strainer cap and mixed immediately before flow cytometry. For experiments employing different buffer combinations, the above protocol was identical, with SET used in place of PBS as indicated in Figure 6G: PBS represents the standard assay; PBSSET represents the standard assay but with the mixing, 3rd wash, and flow cytometry conducted in SET instead of PBS; and SET employs SET instead of PBS for all steps. For fluorescence mixture assays, we observed some variability in the calcein-labeling efficiency across samples. Since only fluorescent particles are eligible to report on coincidence or aggregation, we normalize each fluorescent event type (i.e., single-positive/doublepositive) to all fluorescent events within each sample.

\section{Immunostaining}

Crude nuclear pellets were resuspended in blocking buffer (PBS, pH 7.4 with 5\% BSA) supplemented with 1 $\mu \mathrm{M}$ Hoechst 33342 and blocked on ice for $15 \mathrm{~min}$. Primary antibody staining was conducted in blocking buffer at $4^{\circ} \mathrm{C}$ on a rotator for $30 \mathrm{~min}$. Samples were washed with blocking buffer, centrifuged at $500 \times g$ (Eppendorf 5424R) for $5 \mathrm{~min}$ and resuspended in blocking buffer with goat anti-mouse Alexa Fluor 488 . Secondary staining was $4^{\circ} \mathrm{C}$ on a rotator for $20 \mathrm{~min}$. Samples were again washed with blocking buffer plus $1 \mu \mathrm{M}$ Hoechst 33342 and centrifuged at $500 \times g$ (Eppendorf 5424R) for $5 \mathrm{~min}$, following resuspension in PBS for flow cytometry.

P2 crude synaptosomes were briefly fixed in 4\% paraformaldehyde (PFA) in PBS, pH 7.4 for 15 min at room temperature. After washing in blocking buffer to quench residual PFA, synaptosomes were centrifuged at 10,000 $\times g$ (Eppendorf $5424 \mathrm{R})$ for $5 \mathrm{~min}$ at $4^{\circ} \mathrm{C}$. The P2 pellet was resuspended in blocking buffer $+0.1 \%$ Tween 20 at room temperature for 30 min before addition of primary antibodies. Primary antibody staining was conducted at room temperature for $30 \mathrm{~min}$, followed by two washes in PBS with centrifugation and resuspension. Pellets were resuspended in blocking buffer $+0.1 \%$ Tween 20 with secondary antibodies and stained for $20 \mathrm{~min}$ at room temperature. Samples were washed twice with PBS, and final samples were resuspended in PBS $+1.5 \mu \mathrm{g} / \mathrm{ml}$ FM4-64 for flow cytometry. For the PBS-SET condition, the final wash and flow cytometry were conducted in SET buffer.

\section{Absolute particle count measurements}

P2 samples from dilution series (Extended Data Fig. 2-1C) or from repeated centrifugation experiments (Fig. 8; Extended Data Fig. 8-1) were diluted to a precise final volume $(200-500 \mu l)$ in PBS+FM or SET+FM. A precise volume of resuspended CountBright absolute counting beads was added to each sample. Counting beads were run alone to confirm that they could be gated apart from FM4-64-triggered P2 events, and were gated based on SSC and blue fluorescence (BV421 channel). Particle concentration was calculated for each sample as per the manufacturer's guidelines using the number of FM4-64triggered P2 events, count bead events, bead count of the lot (beads $/ \mu \mathrm{l})$, and bead/sample volumes. For repeated centrifugation experiments (all using indicated relative centrifugal force $(\times g)$ on Eppendorf 5424R), absolute particle counts were calculated based on the total sample volume, and total particle counts in each fraction were determined by back-calculation using the aliquot and sample volumes.

\section{Alexa Fluor 488 MESF calibration}

Quantum Alexa Fluor 488 MESF calibration beads were run on the Influx at the same time and under identical conditions (sample pressure, detector voltages, etc.) as the immunostained samples. MESF calibration beads ( 7 $\mu \mathrm{m})$ could be identified based on SSC with only a slight reduction $(\sim 10 \%)$ in the SSC voltage for this experiment. The five calibration beads (blank and MESF beads 1-4) were run individually to confirm separation by the green fluorescence detector (FITC channel). Beads were first gated based on SSC and then based on green fluorescence histograms (Extended Data Fig. 7-1E) using full width at half-maximum gating as recommended by the manufacturer. The median fluorescence intensity of the calibration beads was used to fit the MESF calibration curve (Extended Data Fig. 7-1E) using the QuickCal V.2.3 template from the manufacturer (Bangs Laboratories, Inc.). The calibration curve was used to calculate the median fluorescence intensity of indirect immunofluorescence [Ms anti-vesicular GABA transporter (VGAT) + antimouse Alexa Fluor 488] in MESF for subsets of gated events (Extended Data Fig. 7-1F). Numerical data are available in Extended Data Figure 7-3.

\section{Beads, chemicals, and antibodies}

Polystyrene (PS) bead standards $(200,500$, and $800 \mathrm{~nm}$ in diameter) with refractive index $\eta=1.59$ and YellowGreen fluorescent label were obtained from Polysciences Inc. (Submicron Bead Calibration kit, catalog number BLI832, Bangs Laboratories Inc.). A bead standard mixture of Yellow-Green fluorescent PS beads with refractive index $\eta=1.59$ (110 and $500 \mathrm{~nm}$ in diameter) and nonfluorescent silica beads with refractive index $\eta=1.43$ $(180,240,300,585,880$, and $1300 \mathrm{~nm}$ in diameter) was obtained from Apogee Flow Systems (product \#1493). Unlabeled PS beads $(2.0 \mu \mathrm{m}$ in diameter) were obtained from Spherotech Inc. (catalog number PP-20-10). CountBright absolute counting beads were obtained from Invitrogen (catalog number C36950). Quantum Alexa Fluor 488 MESF calibration bead kit was obtained from Bangs Laboratories, Inc. (catalog number 488).

The following dyes were obtained from Invitrogen/Invitrogen: Hoechst 33342 (catalog number H3570), FM4-64 (catalog number T13320), calcein AM (catalog number C34852), calcein red-orange AM (catalog number C34851), LIVE/DEAD Fixable Violet amine-reactive dye (catalog number L34955), and MitoTracker Deep Red FM (catalog number M22426). Antibodies used in these studies and their concentrations are summarized in Table 3.

\section{Statistical analyses}

Gated data exported from FCS Express 6 were further processed in Excel and in R. Where indicated, the robust SD (rSD) was calculated using FCS Express 6 software. 
Table 3. List of antibodies

\begin{tabular}{|c|c|c|c|c|c|c|}
\hline \multicolumn{7}{|c|}{ Primary antibody list } \\
\hline Origin & Antigen & & Concentration & Manufacturer & Product number & RRID \\
\hline Mouse & VGAT & & $1: 400$ & Synaptic Systems & 131011 & AB_887872 \\
\hline Rabbit & VMAT2 & & $1: 500$ & Immunostar & 20042 & $A B \_10013884$ \\
\hline \multicolumn{7}{|c|}{ Secondary antibody list } \\
\hline Origin & Antigen & Fluorophore & Concentration & Manufacturer & Product number & RRID \\
\hline Goat & Rabbit $\lg G(H+L)$ & Alexa Fluor 647 & $1: 500$ & Invitrogen & A-21244 & AB_2535812 \\
\hline
\end{tabular}

All graphs were generated using the ggplot2 package in $\mathrm{R}$, except those in Figure 8 and Extended Data Figures $2-1 C, 7-1 C-F, 8-1 A-F$, which were generated in Excel. All statistical testing was conducted in $\mathrm{R}$. Where indicated, a Welch's $t$ test was performed with the indicated contrasts (Figs. 5D, 6D-F; Extended Data Fig. 6-1C). Where indicated, a two-way ANOVA was performed using FSC Gate and Buffer as factors (Figs. $6 H, 7 B, C$ ); degrees of freedom, $F$ statistic, and $p$ values for main effects as well as interaction are listed in the figure captions. A summary of all statistical tests is provided in the statistical table (Table 4).

\section{Results}

\section{FSC and FM4-64 triggering of crude synaptosome fraction P2 and comparison to bead standards}

Conventional flow cytometers generally use FSC measurements for detection of cells, but the sensitivity of each instrument in FSC-triggered detection of submicron particles varies widely depending on the refractive index of the particles, the angle of collection for scattered light, detector sensitivity, and other factors (van der Pol et al., 2013). We used PS and silica beads to determine the relative sensitivity of two cytometers: a BD LSR Fortessa analyzer and a BD Influx sorter. We optimized FSCthreshold parameters on each instrument to maintain a mixture of PS and silica bead standards ranging 585$1300 \mathrm{~nm}$ in the dynamic range of detection, while simultaneously avoiding electronic and buffer noise.

As shown in Figure $1 A$, the lowest FSC signal resolvable above the background noise on the Influx was the 585-nm silica bead. Cross-referencing the FSC signal with the green fluorescence signal allowed us to clearly identify PS beads apart from the non-fluorescent silica beads (Fig. $1 A$, right). As expected due to their higher refractive index, the FSC signals for PS beads were substantially higher than for similarly sized silica beads. We also identified events that appeared to be simultaneous detection of two 500-nm PS beads, which we confirmed by comparing their FSC and green fluorescence to the "singlet" gate as previously described (Extended Data Fig. 1-2; Stoner et al., 2016). Although the FSC and fluorescent signals appeared additive in the 500-nm PS doublet population (1.7-fold higher FSC, 1.8-fold higher green fluorescence), the FSC signal for these events still fell below the $880-\mathrm{nm}$ silica beads. In contrast to the Influx, the Fortessa displayed poor FSC resolution when running the silica/PS bead mixture (Fig. 1B), which required separately running the individual PS beads [500- and 800-nm PS labeled with (B) in Influx plots]. The lowest FSC signal resolvable above the noise background on the Fortessa included $880-\mathrm{nm}$ silica as well as 500-nm PS singlet and "doublet" bead populations, which could only be separated by SSC or green fluorescence intensity (Fig. 1B). A complete summary of FSC and SSC measurements for gated bead populations can be found in Extended Data Figure 1-2.

Having established FSC-triggering limits and relative bead positions on the instruments, we next sought to compare FSC and fluorescence triggering of P2 crude synaptosome (hereafter P2) samples. Despite contamination by a heterogeneous mixture of free mitochondria, myelin, and membranous debris, P2 samples are enriched with synaptosomes and have been widely employed in flow cytometry studies (Gylys et al., 2000; Fein et al., 2008; Postupna et al., 2014; Prieto et al., 2017). As previously described by Biesemann et al. (2014), FM4-64 is a styryl dye with favorable properties for fluorescencetriggered detection of synaptosomes. FM4-64 is minimally fluorescent in aqueous media but becomes intensely fluorescent on partitioning into membranes (Vida and Emr, 1995), and its far-red emission is efficiently excited by a 488nm laser, which is required for triggering on our cytometers. As shown in Figure 1C,D, FM triggering enabled highly sensitive detection of P2 samples compared to FSC triggering, with more than half of particles in the P2 sample below the FSC noise threshold on both cytometers. These results are consistent with the work of Biesemann (2010; his Results 3.5, Fig. 15, pp 87-90), who found $\sim 70 \%$ of particles in sucrose gradient synaptosome preparations were undetectable by FSC triggering on the BD FACS Aria.

Since previous studies have analyzed putative synaptosomal particles with FSC signals as high as 4.5- $\mu \mathrm{m}$ PS beads (Gylys et al., 2000), we sought to assess the size of biological particles in such an FSC range. In the absence of a refractive index mismatch between PS beads and biological particles, one would expect neuronal nuclei, which sediment in the P1 fraction, to exhibit significantly higher FSC than 2- $\mu \mathrm{m}$ PS beads due to their size. Although we had to lower the FSC voltage to place 0.5-, $0.8-$, and $2-\mu \mathrm{m}$ PS beads within the same dynamic range, 2.0- $\mu \mathrm{m}$ PS beads were clearly resolved from $0.5-$ to $0.8-\mu \mathrm{m}$ PS beads by FSC on both the Influx and Fortessa 
Table 4. Statistical table

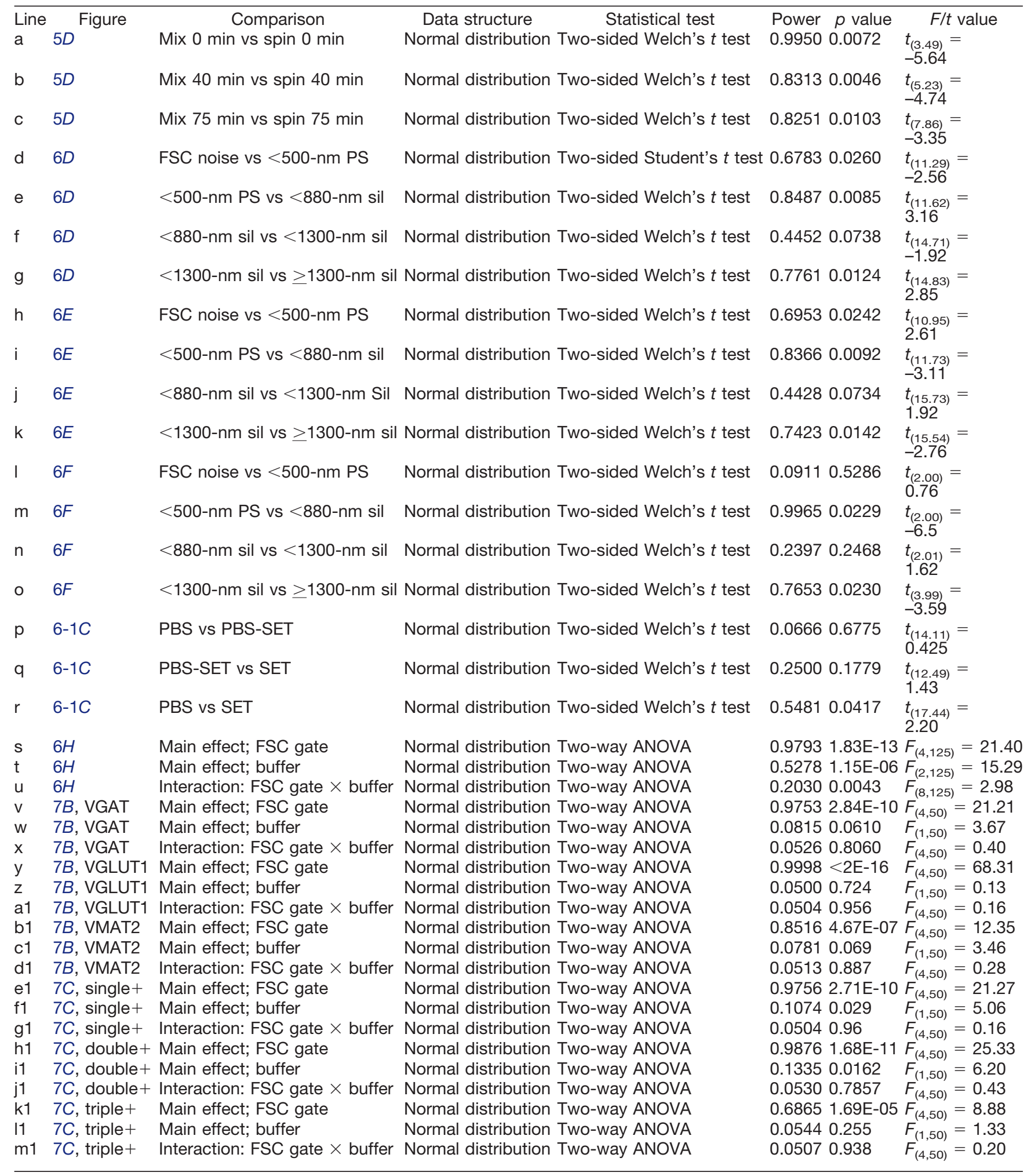

(Extended Data Fig. 1-1A). We identified neuronal nuclei based on Hoechst 33342 intensity and NeuN immunofluorescence (Extended Data Fig. 1-1B) and found that they were adjacent to and partially overlapping with the FSC range defined by $2-\mu \mathrm{m}$ PS beads on the Influx (Extended
Data Fig. 1-1C). These results confirm that FSC-based sizing relative to PS beads underestimates the size of biological particles with lower refractive indices, and suggest that a majority of nuclei would be included in FSC gates containing 4.5- $\mu \mathrm{m}$ PS beads. 
A

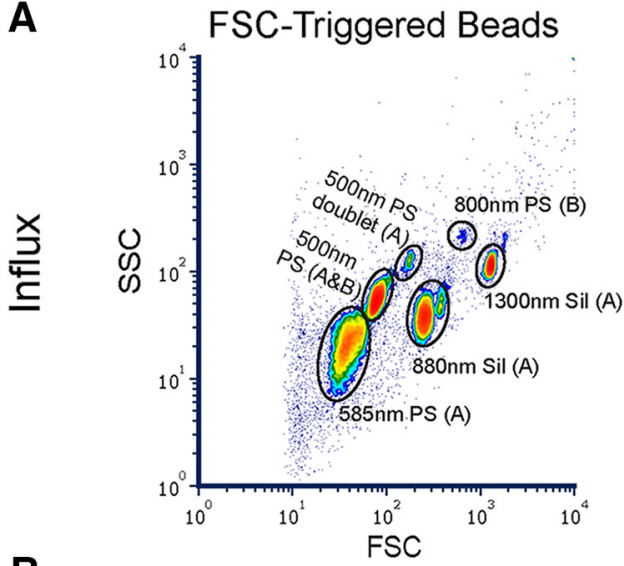

B

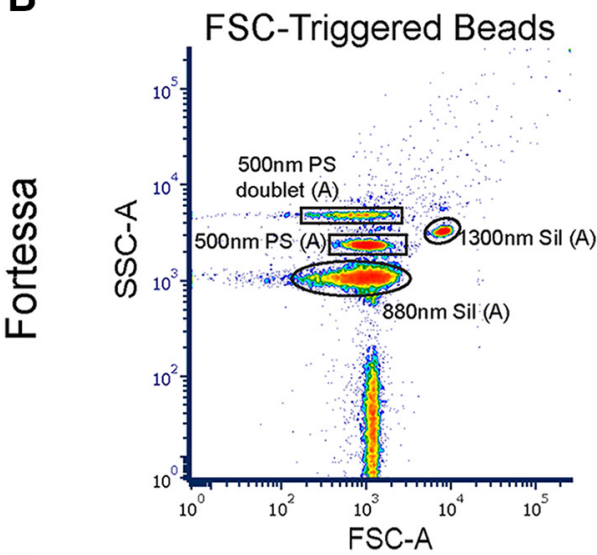

C

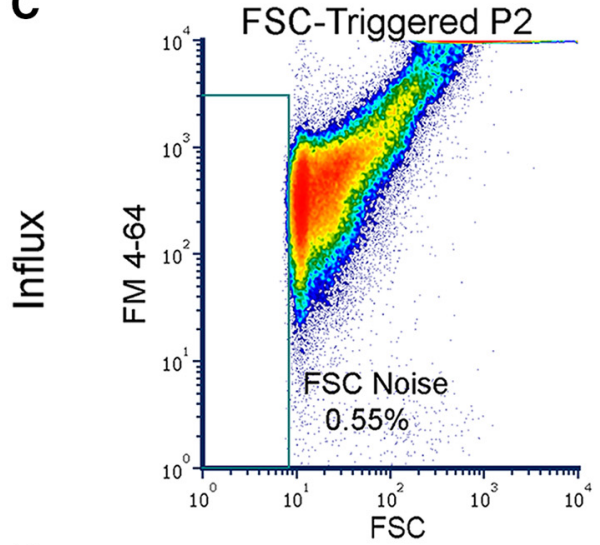

D

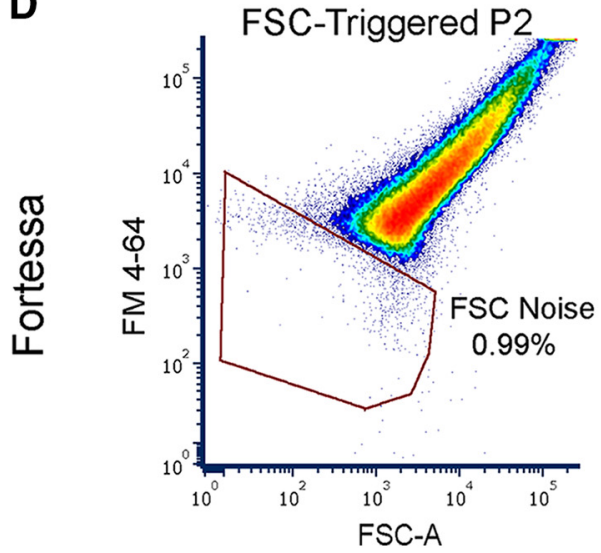

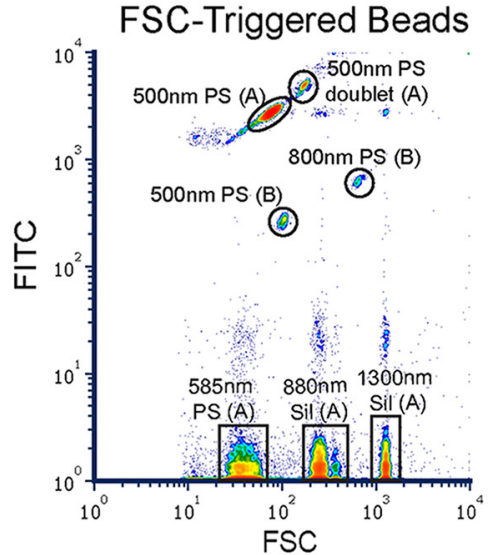
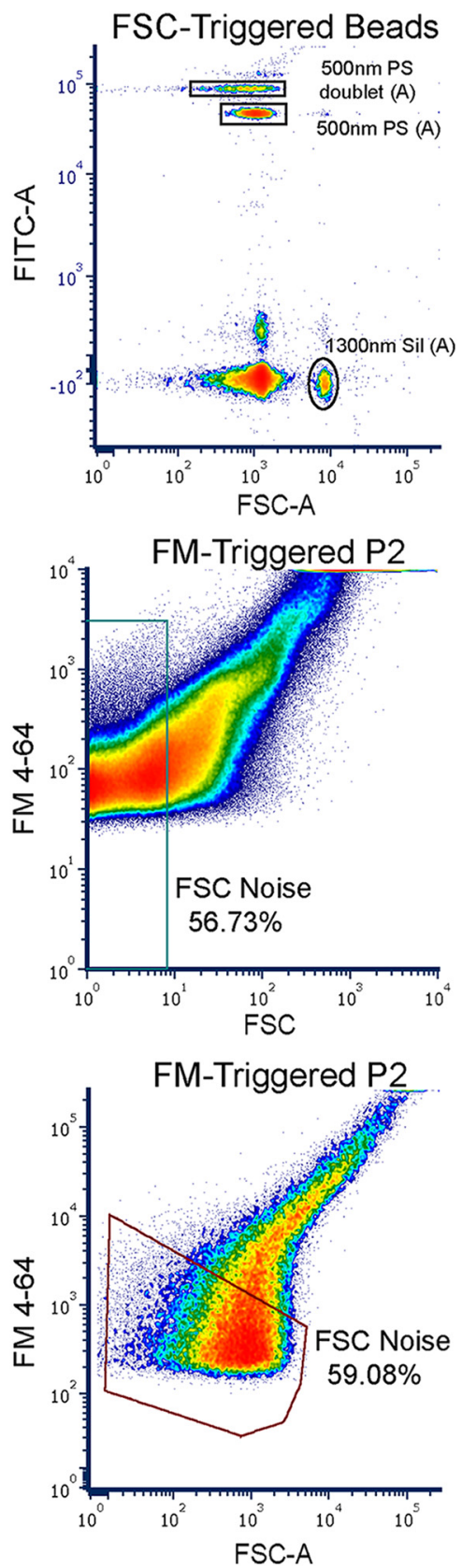

Figure 1. Determining detection sensitivity using bead standards and FSC versus FM triggering of P2 fraction. $\boldsymbol{A}$, Mixture of 
continued

non-fluorescent silica/fluorescent PS beads (Apogee; A) and fluorescent PS beads (Bangs Labs; B) detected in FSC-trigger mode on the Influx. Forward and side scatter (left) FSC (right) and green fluorescence detector (FITC) allows fluorescent PS beads and bead doublets to be clearly distinguished from non-fluorescent silica beads. $\boldsymbol{B}$, Mixture of non-fluorescent silica/fluorescent PS beads (Apogee; A) detected in FSC-trigger mode on the Fortessa. Note that the Bangs Labs 500- and 800-nm fluorescent PS beads could not be run simultaneously with the Apogee mixture on the Fortessa due to poor FSC resolution. C, FM4-64-stained P2 sample on the Influx. Left, FSC-trigger mode. Right, FM-trigger mode. FSC noise gate was set by running clean PBS. D, FM4-64 stained P2 sample on the Fortessa. Left, FSC-trigger mode. Right, FM-trigger mode. FSC noise gate was set by running clean PBS. See Extended Data Figure 1-2 for median FSC and SSC values from gated bead populations. See also Extended Data Figure 1-1.

\section{Serial dilutions define a range of linear detection for FM4-64 triggering}

It has previously been demonstrated that high concentrations of particles below the trigger threshold can be detected when they simultaneously occupy the focal point of illumination (van der Pol et al., 2012; Nolan and Stoner, 2013). This phenomenon of coincident detection (also known as "coincidence" or swarm detection) is thought to be particularly problematic for FSC-triggered detection of submicron particles (Nolan and Stoner, 2013). to define a linear range of particle detection and further compare FSC versus FM triggering, we conducted dilution series on both Influx and Fortessa. Compared to FM triggering, FSC-triggered P2 samples did not maintain a constant FSC versus FM4-64 profile on either the Influx or Fortessa (Fig. 2A,B; Extended Data Fig. 2-1). Specifically, the abundance of events near the FSC threshold with low FM4-64 fluorescence decreased with dilution, and the appearance of "noise" events (although $<10$ events/s) became more apparent with dilution on the Fortessa. On the Influx, quantification of the event rate across the dilution series revealed a sub-linear profile for both FSC and FM triggering, which was fit with high accuracy using a quadratic model $\left(R^{2}=0.99\right.$ for both series; Fig. $2 C$, left panel). FM triggering was similar on the Fortessa, but FSC triggering was better fit with a linear model $\left(R^{2}=0.93\right.$ for FSC and $R^{2}=0.99$ for FM series; Fig. $2 D$, left panel). These results confirm that event rate saturates at high particle concentrations (Kormelink et al., 2016) and that FSC triggering underestimates the true event rate across the entire dilution series.

Given that much of the P2 remains undetected by FSC triggering, we also wondered whether particle concentration might alter the FSC and FM fluorescence measurements. On the Influx, the median FSC remained relatively constant for both FSC and FM triggering (Fig. 2C, middle panel), while the median FM4-64 fluorescence varied dramatically for FSC triggering but not for FM triggering (Fig. $2 \mathrm{C}$, right panel). On the Fortessa, both median FSC and median FM4-64 fluorescence varied dramatically across the dilution series for FSC triggering but not FM triggering (Fig. 2D, middle and right panels). The increase in median FM4-64 fluorescence with dilution seen in FSC-triggered samples seems related to the "FM4-64 low" events described above. Given the heterogeneous population of particles contained in P2 samples, we cannot make strong conclusions about the mechanisms underlying these effects. Nonetheless, it is clear that FM triggering is superior to FSC triggering in terms of the stability and accuracy of event rate, FM4-64 fluorescence, and FSC of
P2 particles across the dilution series on both instruments. We conducted all further studies using only FM triggering and maintaining reasonably dilute samples so as to stay in the most linear range of the event rate dilution curves (fewer than 10,000 events/s on the Influx and fewer than $\sim 3000$ events/s on the Fortessa; Fig. $2 C, D$, left panels). Since sample flow rates and event rates may vary across cytometers, we also measured the absolute particle concentration across a typical dilution series on the Influx using volumetric count beads (Extended Data Fig. 2-1C). We found that the most linear range of the event rate curve was for samples below $\sim 1000$ particles $/ \mu \mathrm{L}$. A previous study on the Influx (Kormelink et al., 2016) showed that the trigger pulse baseline becomes continuously elevated at high event rates where coincidence is prominent, so we used a digital oscilloscope on the Influx to confirm that the trigger pulse remained at baseline under these conditions (data not shown).

\section{Development of a multicolor fluorescence assay for false colocalization}

Although FM triggering and dilution should help to reduce coincident particle detection, another reported source of "false double-positive" events in submicron flow cytometry is the physical aggregation of multiple particles (Erdbrügger et al., 2014). Multiple microscopy studies have noted the presence of aggregated synaptosomes (Gray and Whittaker, 1962; Choi et al., 2009; Daniel et al., 2012), but conventional flow cytometry does not provide a means to verify whether events truly represent single particles. We sought to design a fluorescencebased flow cytometry assay to estimate false doublepositive events in our P2 samples (Fig. 3).

The assay workflow is similar to previously described "cell barcoding" flow cytometry methods (Krutzik and Nolan, 2006; Krutzik et al., 2011). A P2 sample is first split and single-labeled with spectrally separated, nontransferable fluorescent dyes. After washing, singlelabeled samples are mixed before flow cytometry (Fig. $3 A)$. Single particle events should therefore bear only one fluorescent label, while coincidences and aggregates derived from separate single-labeled samples will be positive for multiple fluorescent labels (Fig. 3B). Although the samples are washed twice to remove any residual dye, it is critical to use dyes that cannot be transferred between particles. Synaptosomes are well-labeled with calcein AM dyes (Gylys et al., 2000; Daniel et al., 2012; Prieto et al., 2017), lipophilic dyes that rapidly cross cell membranes and become trapped following hydrolysis of the AM ester. In addition to calcein AM and calcein red, we also used 
A

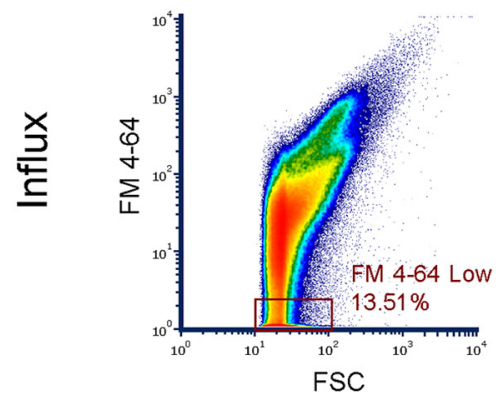

B

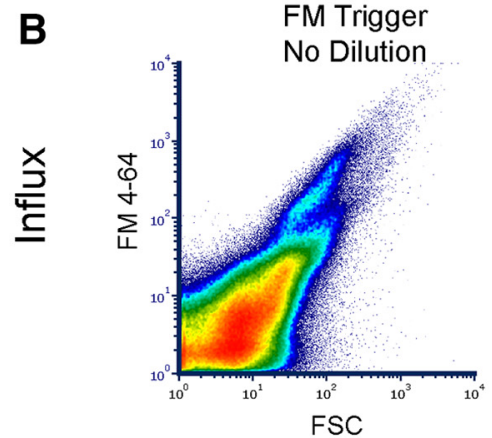

C

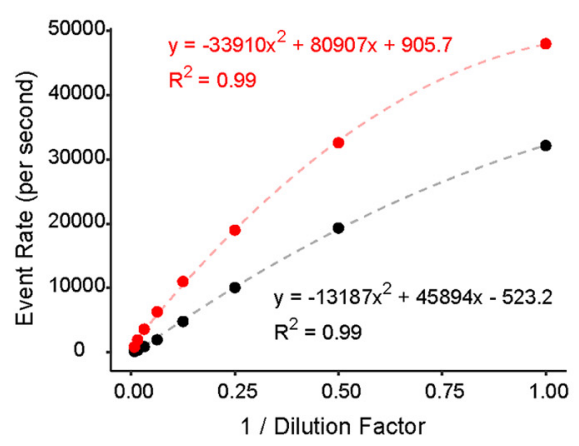

D

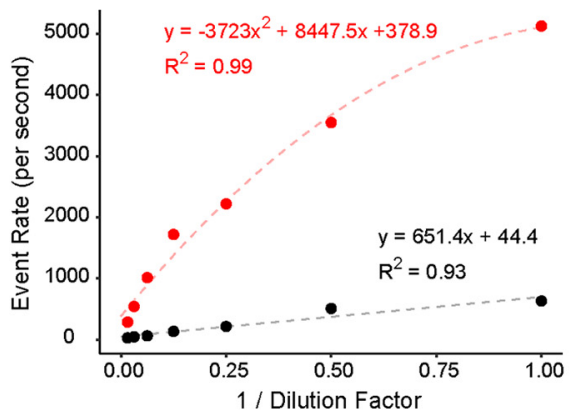

FSC Trigger

Dilution 1:8

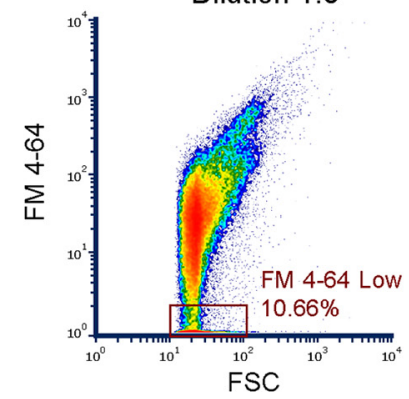

FM Trigger Dilution 1:8

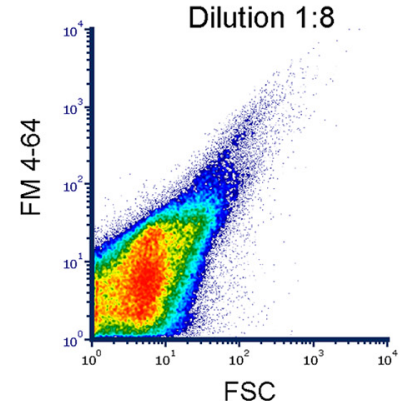

FSC Trigger

Dilution 1:32

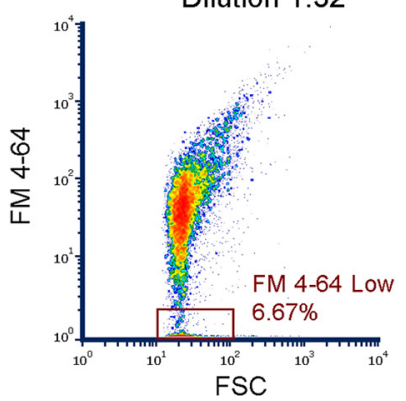

FM Trigger

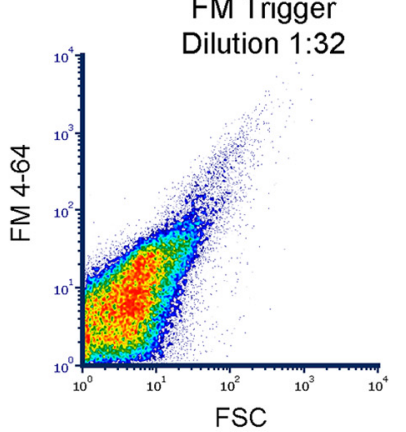

FSC Trigger

Dilution 1:64

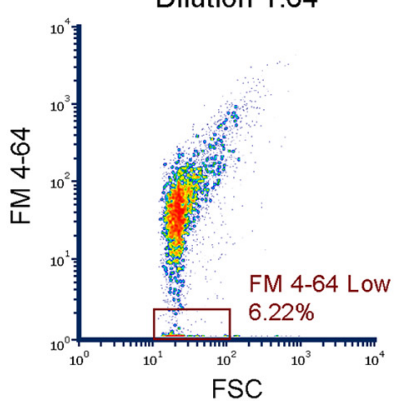

FM Trigger

Dilution 1:64

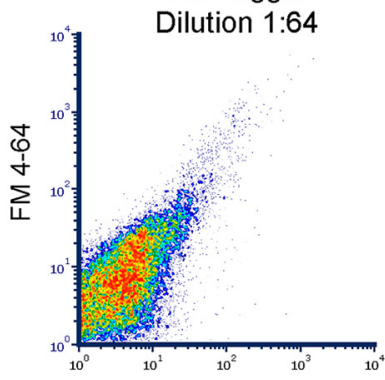

FSC

Influx Dilution Series

- FSC Trigger • FM Trigger
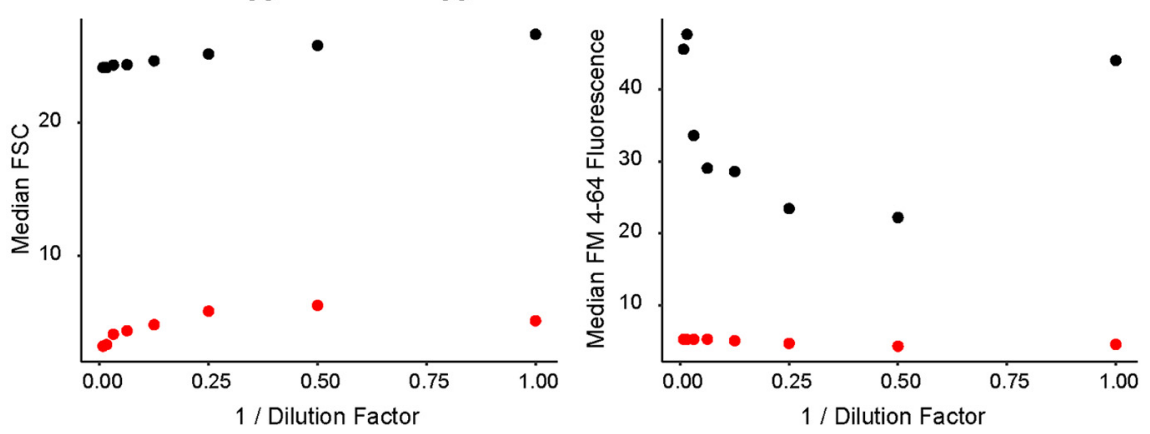

Fortessa Dilution Series

- FSC Trigger • FM Trigger
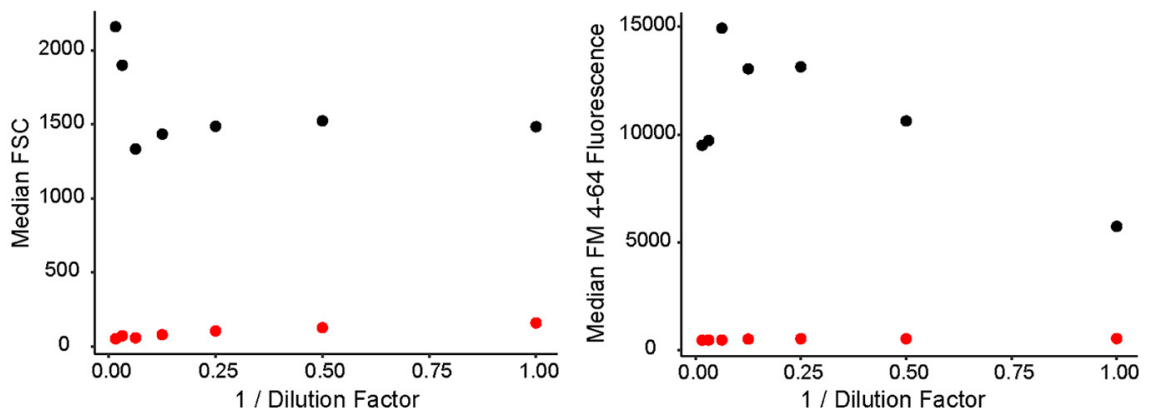

Figure 2. Dilution series define a range of linear particle detection in P2 samples. $\boldsymbol{A}$, Representative density plots of a P2 dilution series detected on the Influx in FSC-trigger mode. FM4-64 low gate was set arbitrarily to quantify disappearance of events in this region with dilution. $\boldsymbol{B}$, Representative density plots of same P2 dilution series as in $\boldsymbol{A}$, but detected on the Influx in FM-trigger mode. $\boldsymbol{C}$, Measurements from full P2 dilution series on the Influx. Left, Event rate data fit with a quadratic model. Middle, Median FSC of all detected events. Right, Median FM4-64 fluorescence of all detected events. $\boldsymbol{D}$, Measurements from full P2 dilution series on the Fortessa. Left, FM-trigger series fit with a quadratic model and FSC-trigger series fit with a linear model. Middle, Median FSC of all detected events. Right, Median FM4-64 fluorescence of all detected events. See also Extended Data Figure 2-1. 


\section{Unlabeled P2 Crude Synaptosome Sample}

Glial/non-synaptic Myelin fragments Synaptosomes membranes

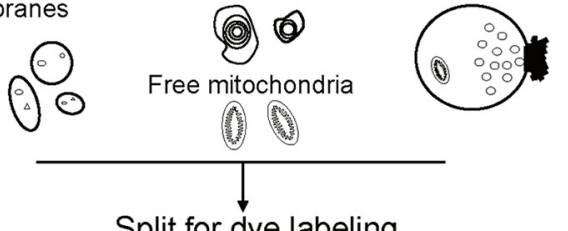

Split for dye labeling
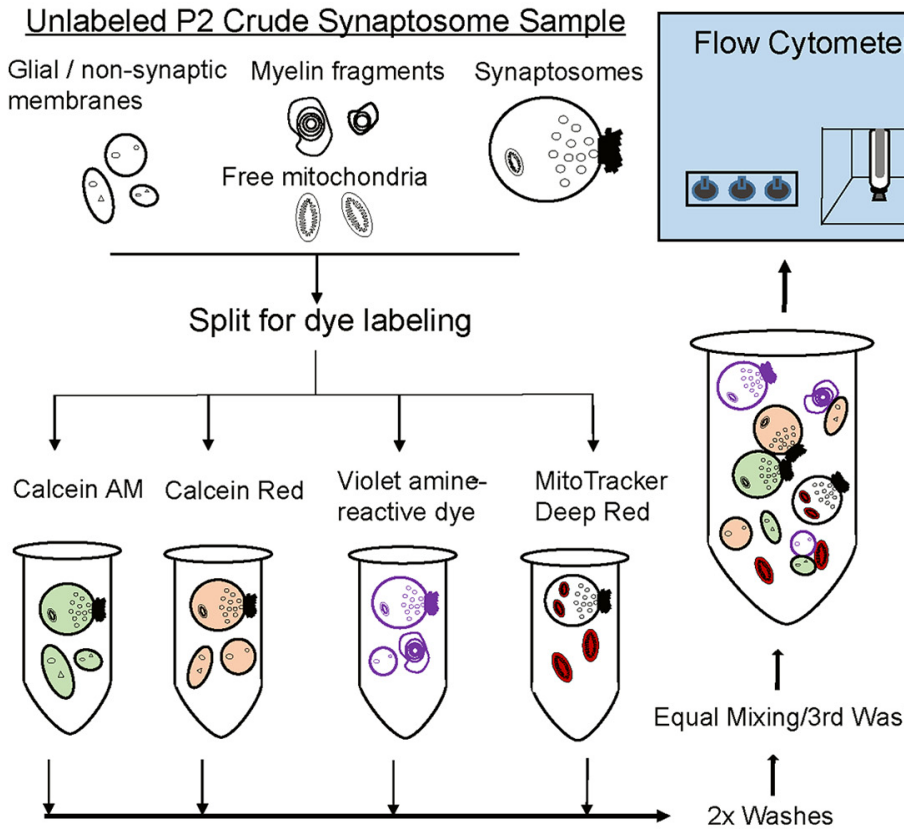

Equal Mixing/3rd Wash

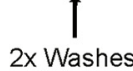

B

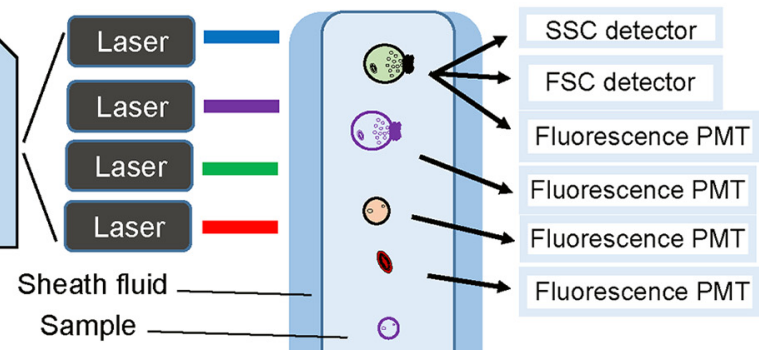

Trigger Parameter

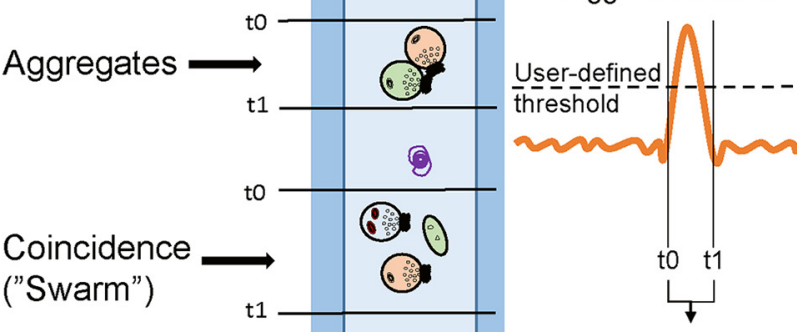

0

Integrate all detectors between $\mathrm{t} 0$ - $\mathrm{t} 1$

$\rightarrow$ "single" event

C

Calcein AM-Labeled P2

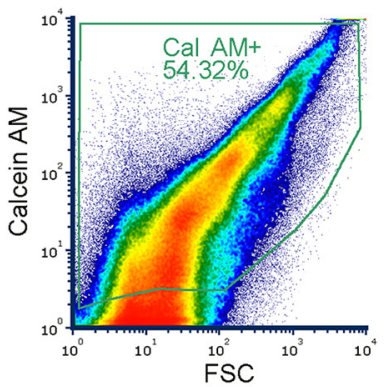

Calcein AM-Labeled P2

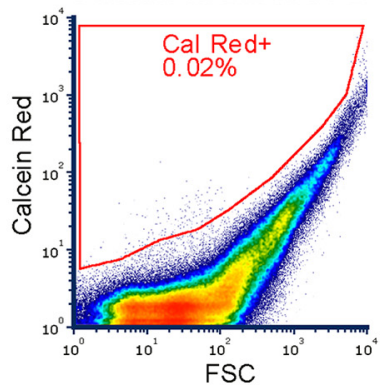

Calcein Red-Labeled P2

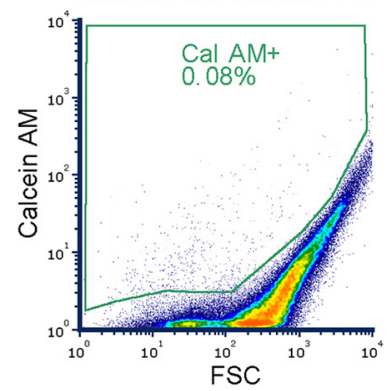

Calcein Red-Labeled P2

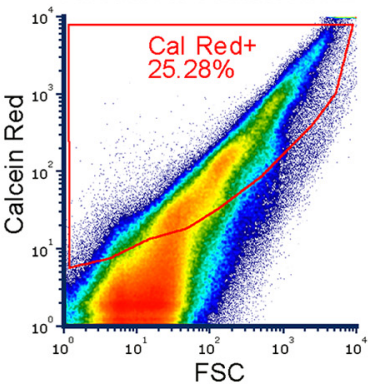

Mix of Single-Labeled P2

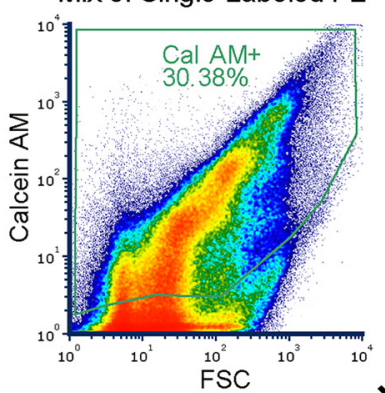

Mix of Single-Labeled P2

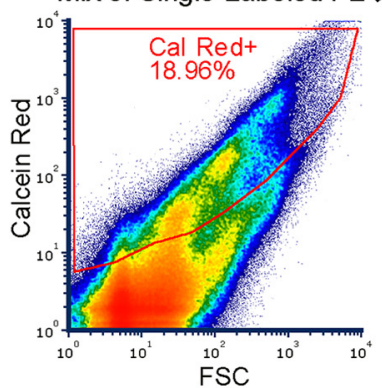

Mix of Single-Labeled P2

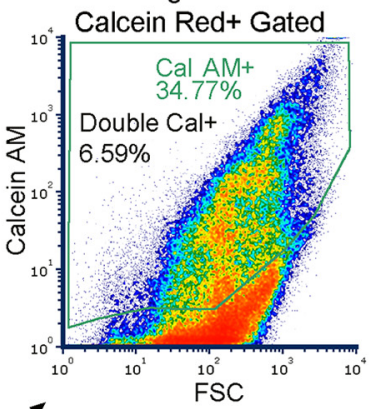

Mix of Single-Labeled P2

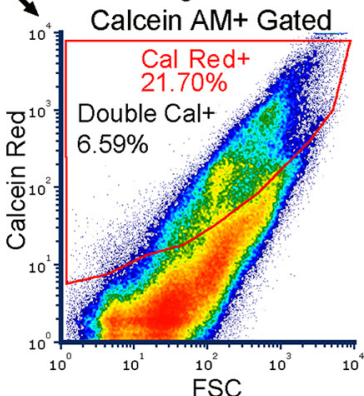

Figure 3. Experimental assay for measuring coincidence and aggregation. A, Schematic representation of the P2 sample labeling workflow. A single sample is split into equal portions for single-color labeling, followed by two washes to remove residual dye. The samples are then mixed and washed a third time before flow cytometry. $\boldsymbol{B}$, Schematic representation of particle detection and artifacts in microparticle flow cytometry. Particles flow in the sample fluid, which is surrounded by sheath fluid. Note that the width of the sample stream is enlarged for illustrative purposes; we run our cytometers at the minimum sample pressure (and thus sample stream width) to ensure optimal hydrodynamic focusing. When the trigger parameter detector crosses a user-defined threshold, the time duration of this pulse is then integrated on all detectors to generate an event. Single events that are detection artifacts occur when multiple particles simultaneously occupy the focal point of laser illumination (coincidence) or are physically bound (aggregates). $\boldsymbol{C}$, Representative density plots of a P2 sample from the double-calcein assay on the Influx. Left, Calcein AM-labeled P2 sample shows no cross-emission in the calcein red detector. Mid-left, Calcein red-labeled P2 sample shows no cross-emission in the calcein AM detector. Mid-right, Mixture of single-labeled P2 samples is gated for calcein AM+ and calcein red+ particles, which are then cross-checked for the other fluorophore (diagonal arrows). Right, Calcein AM+ or calcein red + gated particles are analyzed to 
continued

quantify double calcein + events. See also Extended Data Figure 3-1.

violet amine-reactive dye (covalent reaction with proteins) and MitoTracker Deep Red FM (thiol-conjugation and retention in mitochondria) to expand the number of dyes in some experiments. As shown in Extended Data Figure 3-1A, samples single-labeled with any of the four dyes do not exhibit any spectral overlap.

A representative set of density plots for a $\mathrm{P} 2$ sample single-labeled with calcein AM or calcein red, as well as a mixture of the two, on the Influx is shown in Figure $3 C$. Note that because even the background fluorescence is linearly related to FSC on the log-log plots, quadrantbased gating of double-positive events underestimates the number of positive events. To avoid this problem, we used polygon gating of each fluorophore individually versus FSC (Fig. $3 C$ ). Boolean logic is used to determine the number of double-positive events, which can be visualized by displaying only the positive-gated population of one fluorophore on a plot of FSC versus the other fluorophore (Fig. 3C, right).

Having verified the presence of calcein double-positive events in P2 mixtures (Fig. $3 C$ ), we sought experimental confirmation that the dyes did not transfer between particles. Because only the cleaved, polar dye molecules are retained, we reasoned that even if some membrane disruption and dye leak occurred during mixing and centrifugation, the polar molecules would be unable to label surrounding intact particles bearing the other calcein dye. We confirmed this by sonicating a P2 sample truly double-labeled with both calcein dyes and adding an unlabeled P2 sample to the sonicated solution using the exact same experimental workflow. As shown in Extended Data Figure 3-1B, no calcein AM-positive or calcein red-positive events were detected. This demonstrates that even after complete membrane disruption to liberate the entire dye content of one sample, we do not observe measurable transfer to an unlabeled sample after washing and mixing.

\section{Aggregation, but not coincidence, causes false double-positive events in P2 samples}

With our multicolor fluorescence assay in hand, we turned toward distinguishing between coincidence and aggregation. Similar to previous studies (Libregts et al., 2018), we used mixtures of green- and red-fluorescent $500-n m$ PS beads to model coincidence. We repeated the dilution series experiments with mixtures of beads or mixtures of single calcein-labeled P2 samples on both Influx (Fig. $4 A, B$ ) and Fortessa (Extended Data Fig. $4-1 A, B)$. For bead mixtures, we observed a clear population of double-positive events (red- and green-bead coincidences) on both instruments. At high concentrations, the Fortessa performed particularly poorly with regard to coincidence, with a wide smear of red-fluorescent bead multiplets and double-positive events (Extended Data Fig. 4-1A). On both instruments, we observed a linear reduction in the number of double-positive bead events with dilution, with virtually none detected at event rates $<500$ events/s (Fig. 4C,D). In contrast, the percentage of double-positive events from P2 samples remained relatively constant across the entire dilution series. P2 double-positive events persisted even at event rates $<100$ events/s, which strongly suggests that coincidence is not the source of these false double-positive events (Fig. 4C,D).

We speculated that the double-positive events might come from the physical association of two separate fluorescent particles in the P2 samples. To further probe the nature of these events, we sorted double-positive events from bead or P2 mixtures on the Influx and reanalyzed the sorted material (Fig. 5A,B). Sorting of bead doublepositive events failed to increase the frequency of these events in the sorted sample, with reanalysis showing a $\sim 4$-fold decrease (Fig. 5C). Consistent with the dilution experiments, this result suggests that the majority of green- and red-fluorescent beads are not physically associated with each other during detection of doublepositive events. In contrast, sorting of P2 double-positive events increased the frequency of these events $\sim 3$-fold in the sorted sample reanalysis (Fig. $5 \mathrm{C}$ ). We note that a 3 -fold increase in fluorescent particles during sort reanalysis is similar to that achieved by (Biesemann et al., 2014) for fluorescent VGLUT1 + synaptosomes, suggesting that P2 double-positive events behave as stable, "single particles" in this experiment. It is also possible that the high pressures encountered during sorting physically disrupt some aggregates, as suggested by Biesemann (2010; his Results 3.2, Fig. 12, pp 79-81), which would further decrease the frequency of double-positive events in reanalysis.

The divergent behavior of bead and P2 samples in dilution and sorting experiments strongly suggests that the majority of P2 double-positive events are aggregates. Since we routinely vortex samples at high speed for $5 \mathrm{~s}$ before flow cytometry, this suggests that the doublepositive aggregates are relatively stable. We hypothesized that these aggregates form during centrifugation and are not fully disrupted during resuspension and filtration. To test this hypothesis, we conducted the multicolor dyelabeling assay with and without centrifuging the sample mixture and monitored the samples over time on the Influx. As shown in Figure 5D, centrifugation resuspension significantly increased the frequency of double-positive events compared to mixing without centrifugation. Intriguingly, the frequency of double-positive events increased over time for nearly every sample (Fig. $5 D$, left panel).

Taken together with the dilution and sorting experiments, these results suggest that particle aggregation in $\mathrm{P} 2$ samples is an ongoing process that is accelerated by centrifugation. We emphasize that our assay actually underestimates the frequency of true aggregates; greengreen or red-red aggregates are not detected as doublepositive events, but such aggregates are surely formed 
A

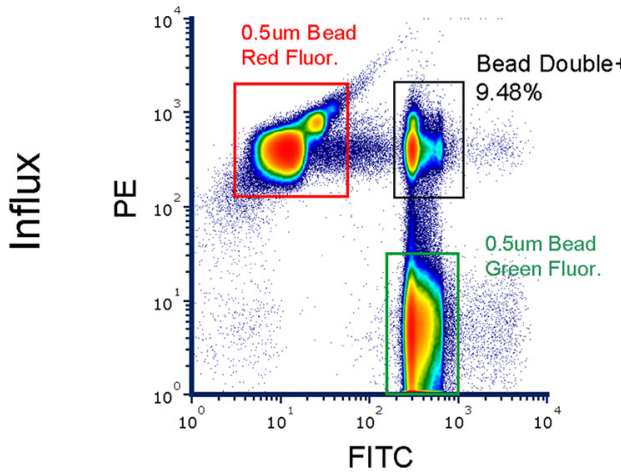

B Mix of Single-Labeled P2 (1:1)

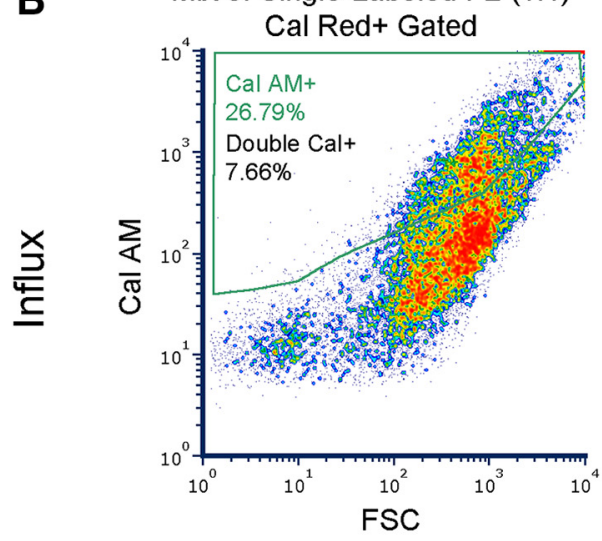

C

D

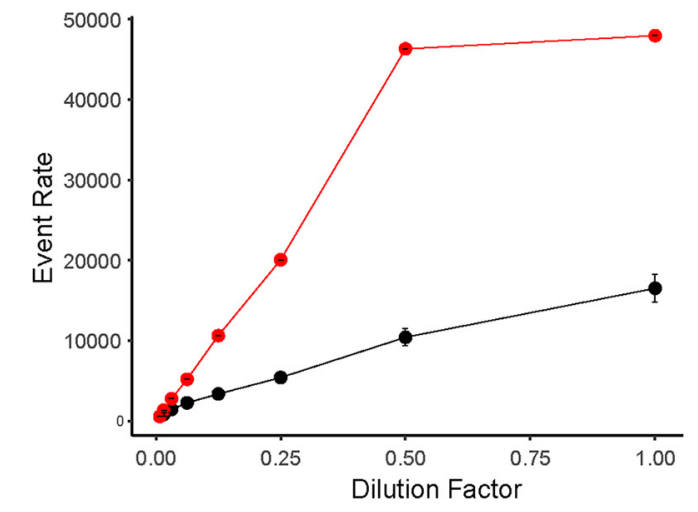

$0.5 \mu \mathrm{m}$ Bead Mixture (1:8)

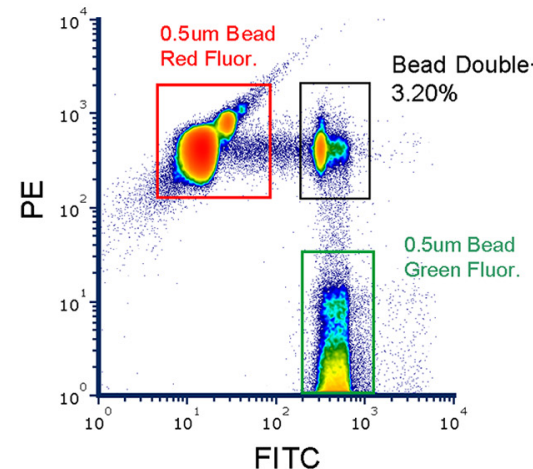

Mix of Single-Labeled P2 (1:4)
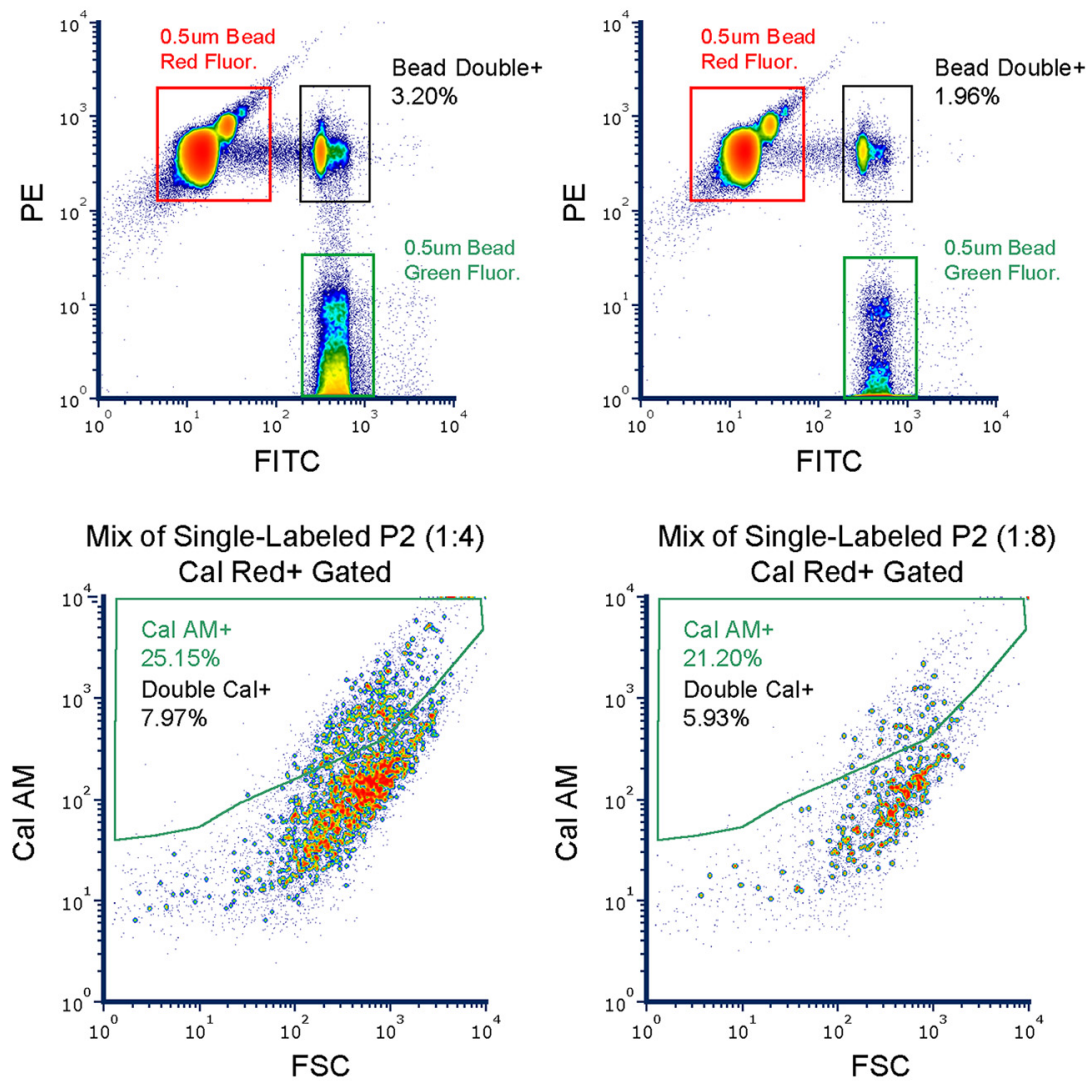

Mix of Single-Labeled P2 (1:8)

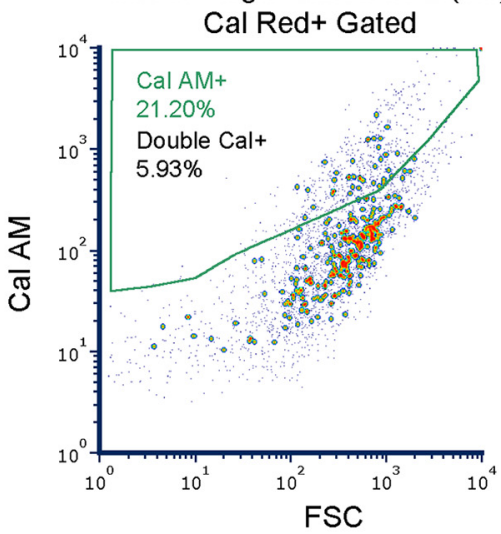

Influx Dilution Series

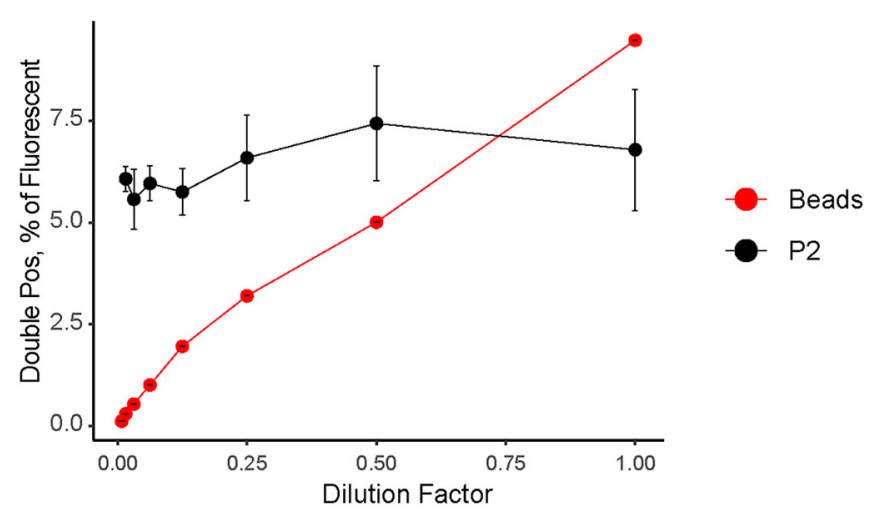

Fortessa Dilution Series
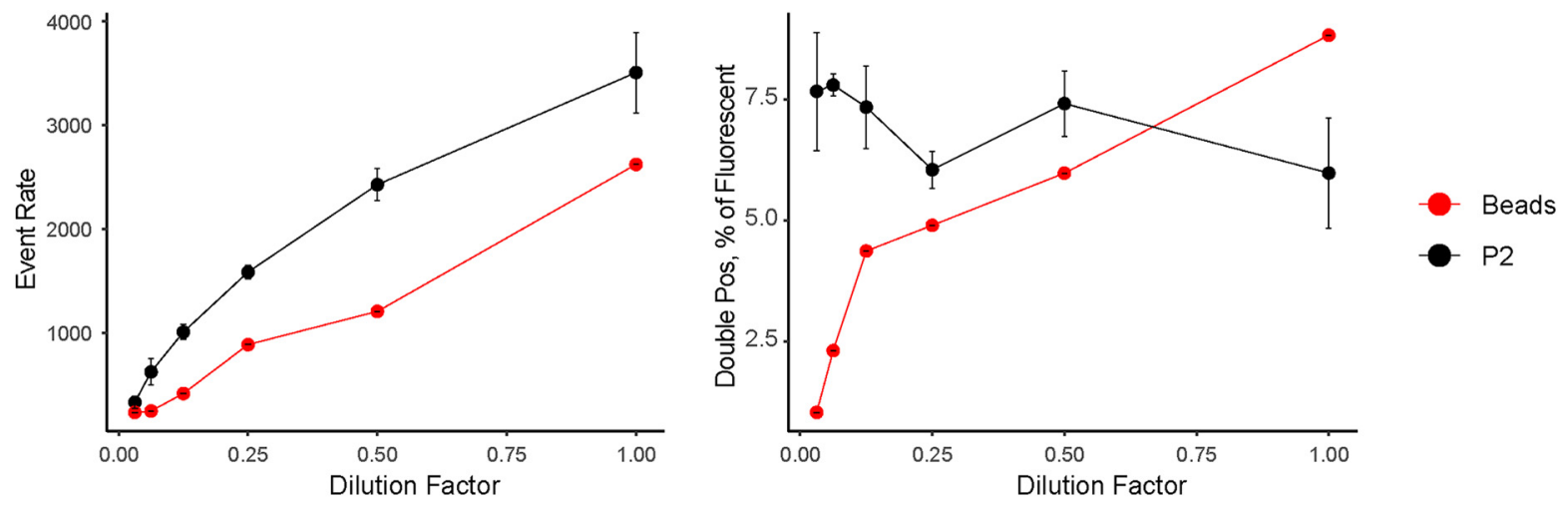
Figure 4. Dilution series reduces false double-positive bead events but not double-calcein positive P2 events. $\boldsymbol{A}$, Representative density plots of a dilution series for a mixture of fluorescent $0.5-\mu \mathrm{m}$ PS beads detected using FSC triggering on the Influx. Individual PS beads and double-positive events are distinguished by their green (FITC detector) or red (PE detector) fluorescence. $\boldsymbol{B}$, Representative density plots of a dilution series for a mixture of single calcein-labeled P2 samples detected using FM triggering on the Influx. Only events gated positive for calcein red are displayed. $\boldsymbol{C}$, Measurements from full dilution series of bead mixture or single calcein-labeled P2 mixtures $(n=3)$ on the Influx. P2 data are displayed as mean \pm SEM. Left, Event rate. Right, Double-positive events expressed as a percentage of all fluorescent events. $\boldsymbol{D}$, Measurements from full dilution series of bead or single calcein-labeled P2 mixtures on the Fortessa. Left, Event rate. Right, Double-positive events expressed as a percentage of all fluorescent events. See also Extended Data Figure 4-1.

during centrifugation and washing of single-labeled samples. The mixing of single-labeled samples without centrifugation shown in Figure $5 D$, mix samples, actually underestimates the true proportion of aggregates, and we therefore conducted all other experiments in this study with centrifugation of the mixtures.

Aggregation of cells can also affect conventional flow cytometry experiments, and we wondered whether commonly employed "singlet gating" procedures could discriminate aggregates in our P2 samples. We plotted the trigger pulse width (in this case, FM4-64) versus FSC on the Influx, or FSC-H versus FSC-A on the Fortessa, for events gated as single or double calcein-positive (Extended Data Fig. 5-1). Cell doublets generally deviate from linearity on such plots, forming a "cloud" that can be gated apart from the linear singlet profile (Wersto et al., 2001). We found no such deviation from linearity in singlepositive gated events, which represent the majority of the sample. Although they bear only one fluorophore, singlepositive events are expected to contain at least as many or even more aggregates than double-positive gated events (i.e., green-green, red-red, red-nonfluorescent, green-nonfluorescent, etc.). The lack of deviation from linearity for any events in these pulse profiles suggests that a "doublet profile" does not exist for our P2 samples. Indeed, a recent flow cytometry study using this gating procedure in analysis of FSC-triggered P2 samples found that all their events obeyed this "linear singlet profile" (Prieto et al., 2017). The lack of a distinguishing doublet profile becomes especially apparent when looking only at double-positive events, which are predominantly aggregates and display a similar linear pulse profile to singlepositive events (Extended Data Fig. 5-1). Thus, we found no evidence that pulse profile is an effective means of removing false double-positive aggregates from downstream analysis of submicron particles.

\section{Double-positive event frequency increases with FSC and is reduced in nonionic buffer}

Although bead standards do not provide accurate size estimation of biological particles, we wondered whether the relative size information afforded by FSC intensity would correlate with the presumably larger size of aggregates. We analyzed five discrete regions ranging from low to high FSC on the Influx, based on beads and the FSC noise threshold (Fig. $6 A, B$ ). A representative overlay of the FSC gates onto gated double-positive $\mathrm{P} 2$ events is shown in Figure 6C. Strikingly, the top two FSC gates contain $\sim 50 \%$ of all double-positive events (percentages in red) but only $\sim 19 \%$ of all calcein-positive events (percentages in black), while the bottom two FSC gates contain only $\sim 25 \%$ of all double-positive events among $\sim 65 \%$ of all calcein-positive events. Accordingly, the percentage of fluorescent events within each FSC gate that were double-positive steadily increased with FSC (Fig. 6C, percentages in blue). For all fluorescent events within each FSC gate, we quantified the percentage of single-, double-, and triple-positive events on the Influx (Fig. $6 D-F)$. Although the overall frequency of single-positives was $\sim 90 \%$, this steadily decreased from $\sim 97 \%$ in the FSC Noise region to $\sim 70 \%$ in the region above or equal to $1300-\mathrm{nm}$ silica beads (Fig. 6D). The overall frequency of double-positives was $\sim 10 \%$, but steadily increased from $\sim 2 \%$ in the FSC Noise region to $\sim 27 \%$ in the region above or equal to $1300-\mathrm{nm}$ silica beads (Fig. 6E). Similarly, the overall frequency of triple-positives was $\sim 2 \%$, but increased from $\sim 0 \%$ in the FSC noise range to $\sim 12 \%$ in the region above or equal to $1300-\mathrm{nm}$ silica beads (Fig. $6 F$ ). These results suggest that although aggregates are present in all FSC regions, their presence is strongly correlated with increasing FSC. Again, we emphasize that our fluorescence assay underestimates aggregation, even in samples where the measured double-positive frequency exceeds $40 \%$ in the highest FSC regions (Fig. 6 C).

Several groups familiar with microscopic analysis of synaptosomal preparations have noted that ionic buffers (e.g., PBS) cause synaptosomes to aggregate, while nonionic media (e.g., sucrose buffer) favors an even distribution of single particles (Choi et al., 2009; Daniel et al., 2012). We repeated our double calcein assay on the Influx using the SET buffer recommended by Daniel et al. (2012). Because many assays are not possible in nonionic media, we tested labeling in PBS and then switching to SET for flow cytometry (designated PBS-SET) in addition to conducting the entire procedure in SET (Fig. 6G). Compared to PBS, both PBS-SET and SET samples displayed similar FSC versus FM4-64 profiles (Extended Data Fig. 6-1A), indicating that SET did not impair FM-triggered particle detection or FSC measurement. FSC and greenfluorescence measurements of PS beads in PBS and SET were also indistinguishable (Extended Data Fig. 6-1B). However, we noticed that the total number of calceinpositive events was reduced to $\sim 20 \%$ in when calcein labeling was conducted in SET, compared to $\sim 40 \%$ for PBS or PBS-SET (Extended Data Fig. 6-1C). We suspect that the nonionic media and divalent cation sequestration by EDTA somewhat reduce the esterase activity of synaptosomes; nonetheless, the labeling efficiency was sufficient to analyze a large number of labeled particles present in these mixtures. As shown in Figure $6 H$, the percentage of fluorescent events within each FSC gate that were double-positive was reduced in SET compared 
A

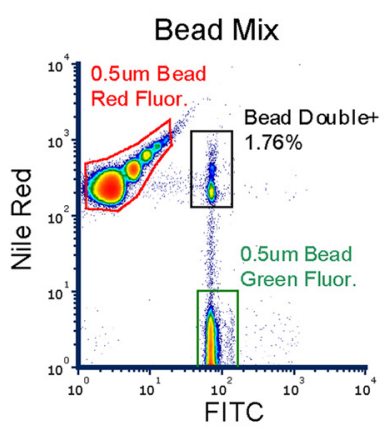

B

\section{Sorted Bead Double+}

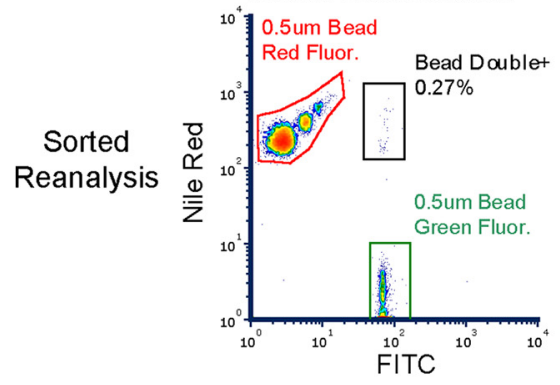

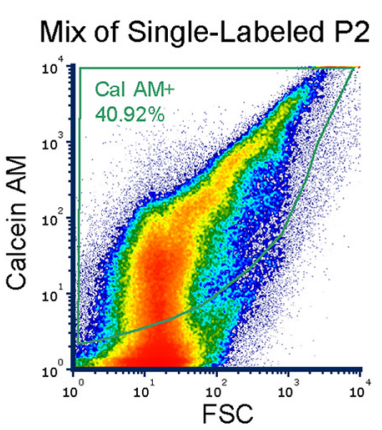

Sorted Double Cal+ P2

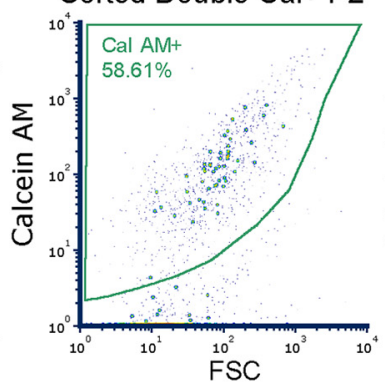

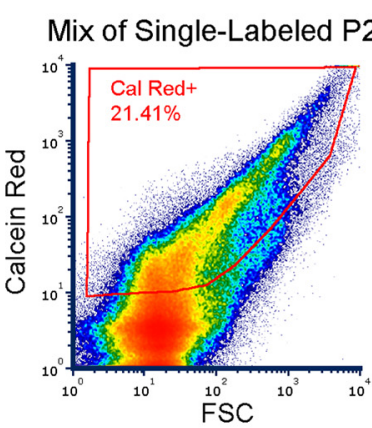

Sorted Double Cal+ P2

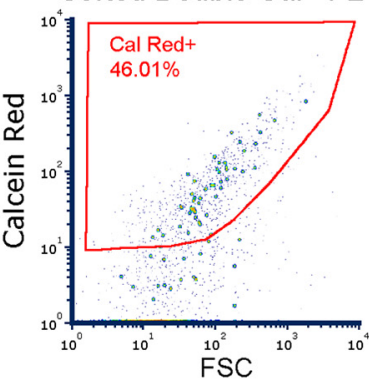

Mix of Single-Labeled P2

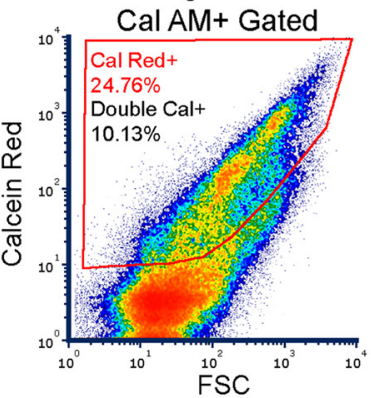

Sorted Double Cal+ P2 Cal AM+ Gated

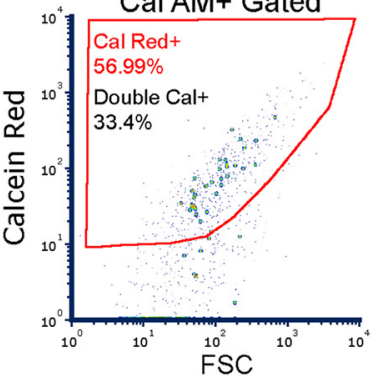

C
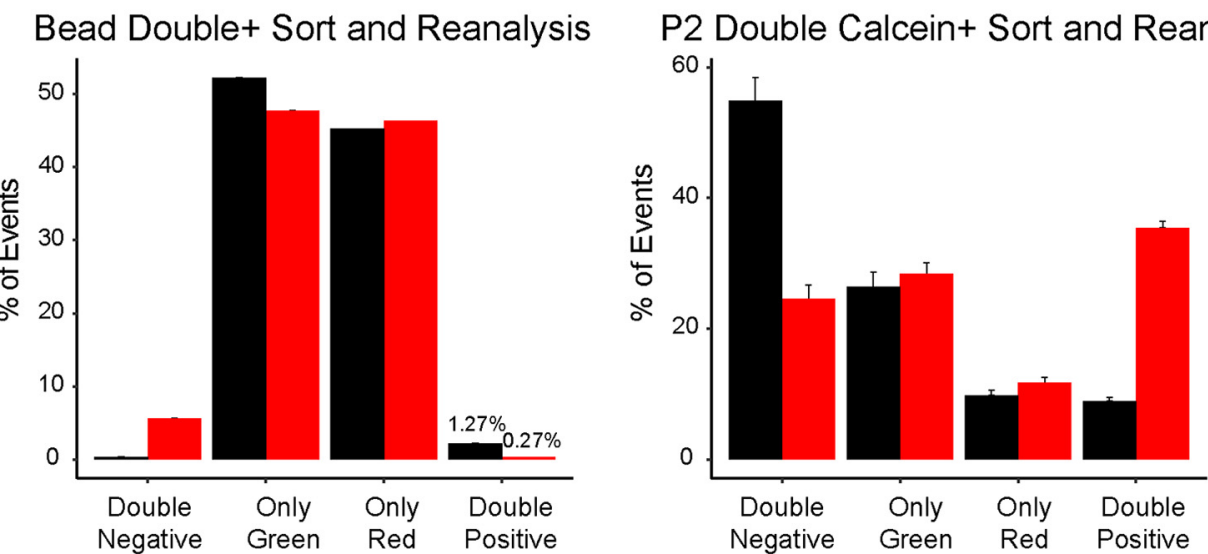

Sample

Pre-Sort Sorted-Reanalysis

Time Lapse After Mix/Spin of Single-Labeled P2

D

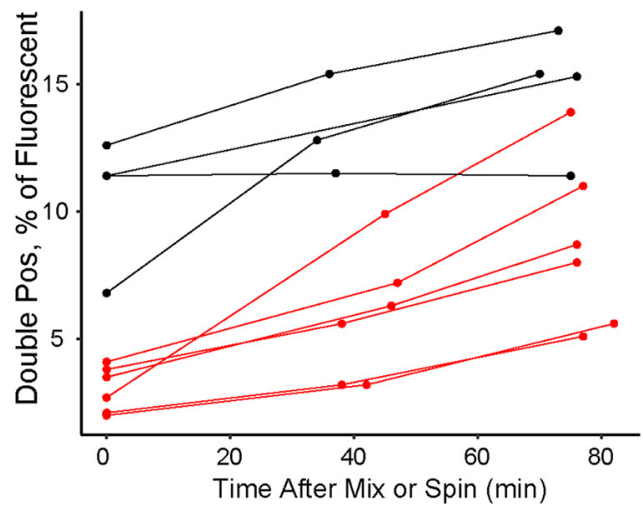

Figure 5. Effects of fluorescence-activated sorting, centrifugation resuspension, and time lapse on double-positive P2 events. These experiments were conducted using FM triggering on the Influx. A, Representative density plots of pre-sorted samples. Left, Mixture of fluorescent $0.5-\mu \mathrm{m}$ PS beads on the Influx. The "bead double+" gate was used for sorting. Mid-left and right, Mixture of single calcein-labeled P2 samples. Right, Calcein AM+ gated events from single calcein-labeled P2 samples were gated for calcein red+. This "double cal+" gate was used for sorting. B, Representative density plots of sorted samples re-analyzed on the Influx. Left, 


\section{continued}

Sample sorted for bead double + gate above. Mid-left and right, Sample sorted for double cal + gate above. Right, Calcein AM+ gated events from double cal+ sorted sample. $\boldsymbol{C}$, Summary of pre-sort versus reanalysis of sorted double-positive bead and P2 $(n=3)$ samples. P2 data are plotted as mean \pm SEM. $\boldsymbol{D}$, Comparison of time lapse data for centrifugation-resuspension ("spin") versus mixing without centrifugation ("mix") of single-labeled P2 samples. Left, Time lapse of double-positive events plotted expressed as a percentage of all fluorescent events. Samples include the following mixtures of single-labeled P2 samples: violet amine-reactive dye/MitoTracker Deep Red FM $(n=2)$, calcein AM/calcein red $(n=3)$, violet amine-reactive dye/calcein AM $(n=1)$, violet amine-reactive dye/calcein AM/calcein red $(n=2)$, all four dyes $(n=2)$. Right, Comparison of averages for the individual trajectories shown on the left, plotted as mean \pm SEM. A Student's $t$ test was performed comparing mix versus spin at each time point; $* p<$ $0.05, * * p<0.01$. See also Extended Data Figure 5-1.

to PBS. A stepwise pattern was observed, wherein the double-positive frequency was highest for PBS, lower for PBS-SET, and lowest for SET. Two-way ANOVA revealed highly significant main effects of both $\mathrm{FSC}$ gate (Df $=5, F$ $=20.06, p=2.7 \mathrm{e}-15)$ and buffer $(\mathrm{Df}=2, F=20.14, p=$ $1.8 \mathrm{e}-8)$ as well as a significant interaction (Df $=10, F=$ $2.78, p=0.004)$. The significant interaction makes sense given that buffer has little effect in the lower FSC regions, while the reduction of double-positive events is greater in the higher FSC regions (Fig. $6 H$ ). These results provide further evidence that double-positive events are aggregates, and that such aggregates are less abundant in nonionic SET buffer.

\section{False colocalization of antigens in immunostained P2 samples}

Given reports claiming high purity of synaptosomes in FSC ranges between 500- to 1500-nm PS beads (Gylys et al., 2004; Postupna et al., 2014; Prieto et al., 2017), we suspected that many of the aggregates in our P2 samples contain synaptosomes. Although the violet amine reactive and calcein dyes efficiently label many particles in P2 samples, they do not provide information about the identity of these particles. We conducted three-color immunostaining of P2 samples on the Influx, targeting presynaptic markers expressed by excitatory, inhibitory, and monoaminergic neurons, respectively: VGLUT1, VGAT, and vesicular monoamine transporter 2 (VMAT2). Although we did not attempt immunostaining in SET buffer, we did mimic the PBS-SET condition described above by resuspending stained and washed samples in SET for flow cytometry. As shown in Figure $7 A$, immunopositive events for all three transporters were observed across the entire FSC range and reflect their abundance in the brain (VGLUT1 > VGAT > VMAT2). Looking at all immunopositive events across FSC gates (Extended Data Fig. 7-1A), VGAT+ and VMAT2 + events displayed an asymmetric distribution centered below 500-nm PS beads, while VGLUT1 + events were distributed fairly uniformly just below $880-\mathrm{nm}$ silica beads. Although the distribution of all immunopositive events across FSC gates was not affected by buffer (Extended Data Fig. 7-1B), the percentage of immunopositive events within each FSC gate was slightly reduced in PBS-SET samples (Fig. 7B). Two-way ANOVA revealed a significant main effect of buffer for VGAT (Df $=1, F=4.70, p=0.034)$ and VMAT2 (Df $=1$, $F=5.21, p=0.026$ ) but not VGLUT1 (Df $=1, F=0.36$, $p=0.55$ ). A highly significant main effect of FSC gate was observed for all three markers $(\mathrm{Df}=5, F=21.51, p=$ 2.9e-12 for VGAT; Df $=5, F=68.9, p<2 \mathrm{e}-16$ for VGLUT1; $D f=5, F=12.54, p=2.4 \mathrm{e}-8$ for VMAT2), where the percentage of immunopositive events within each FSC gate increased with FSC (Fig. 7B). All buffer $\times$ FSC gate interactions were non-significant. Consistent with recent studies (Prieto et al., 2017), the percentage of VGLUT1+ events in the highest FSC gate approaches $\sim 60 \%$.

Although specific cases of neurotransmitter cotransmission and co-release have been observed (for review, see Hnasko and Edwards, 2012; Vaaga et al., 2014), the expression of VGLUT1, VGAT, and VMAT2 should be mutually exclusive for the majority of presynaptic terminals. For all immunopositive events within each FSC gate, we quantified the percentage of single-, double-, and triple-positive events (Fig. 7C). Similar to double calceinpositive events, we observed a steady decrease in singlepositive and a steady increase in double-positive and triple-positive events with increasing FSC. Two-way ANOVA revealed a significant main effect of FSC gate for each event type $(\mathrm{Df}=5, F=19.83, p=1.3 \mathrm{e}-11$ for single-positive; $\mathrm{Df}=5, F=23.25, p=6.6 \mathrm{e}-13$ for doublepositive; $\mathrm{Df}=5, F=8.37, p=4.7 \mathrm{e}-6$ for triple-positive). PBS-SET samples had higher single-positive event frequencies and lower double-positive event frequencies compared to PBS (Fig. 7C). The main effect of buffer was significant for single-positive ( $\mathrm{Df}=1, F=6.74, p=0.012$ ) and double-positive ( $\mathrm{Df}=1, F=8.00, p=0.006$ ), but not for triple-positive events ( $\mathrm{Df}=1, F=1.91, p=0.17$ ). None of the buffer $\times$ FSC gate interactions were significant. Thus, although PBS-SET samples did display reduced aggregation, the effect was modest, especially for triple-positive events. Similar to the percentage of immunopositive events within each FSC gate, the frequency of false co-localization increases with FSC. To further illustrate this point, we calculated the percentage of immunopositive events for each antigen that were single-, double-, or triple-positive within each FSC gate (Extended Data Fig. 7-2). In the FSC noise region we found that $\sim 80 \%$ of VGAT,$+ \sim 75 \%$ of VGLUT $1+$, and $\sim 63 \%$ of VMAT2 + events were single-positive. Strikingly, in the highest FSC gate, the single-positive percentage was only $\sim 12 \%$ for VGAT +, $\sim 51 \%$ for VGLUT1+, and $\sim 9 \%$ for VMAT2. These results suggest that many of the putative "synaptosomal" events in the upper FSC regions are aggregates containing multiple synaptosomes.

The quantification of double-positive events described above represents the sum of the three possible subtypes (i.e., VGAT+NGLUT1+, VGLUT1+NMAT2+, and 
A

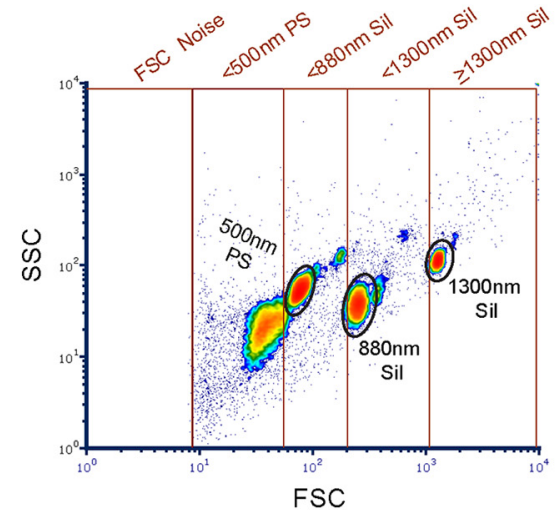

D

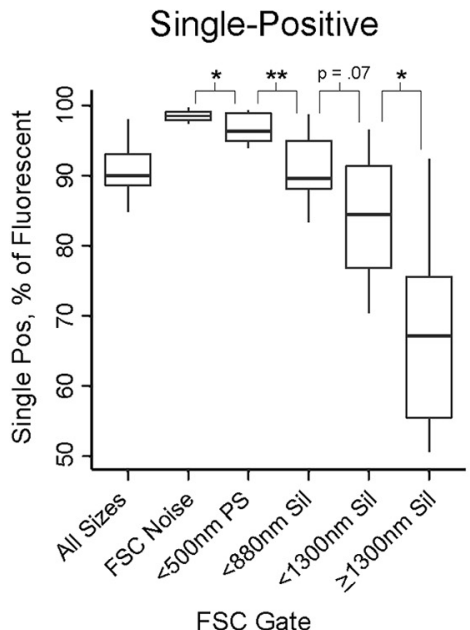

G

Buffer Scheme

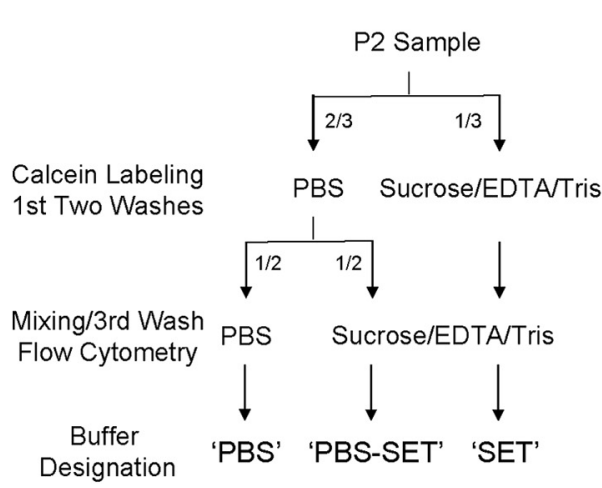

B FSC-Triggered Beads

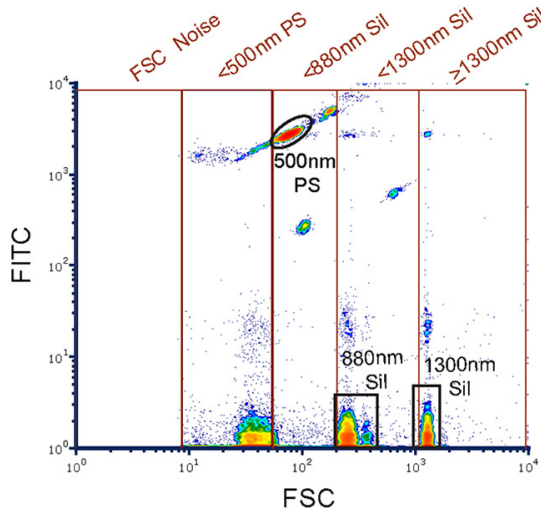

E

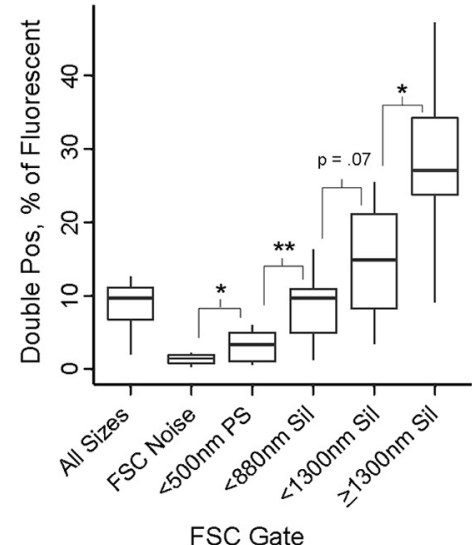

H

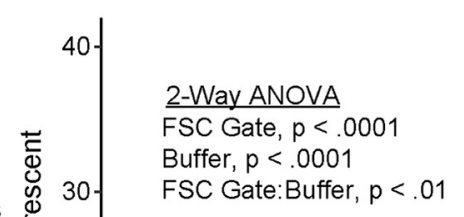

C Mix of Single-Labeled P2

Gated Double Cal+

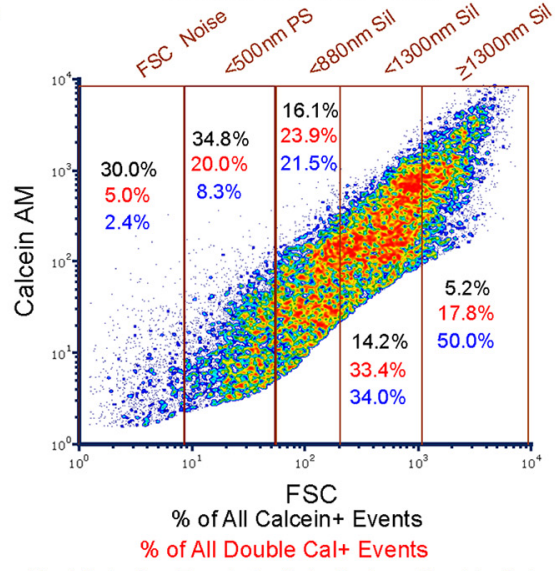

$\%$ of Calcein+ Events in Gate that are Double Cal+

$\mathbf{F}$

Triple-Positive

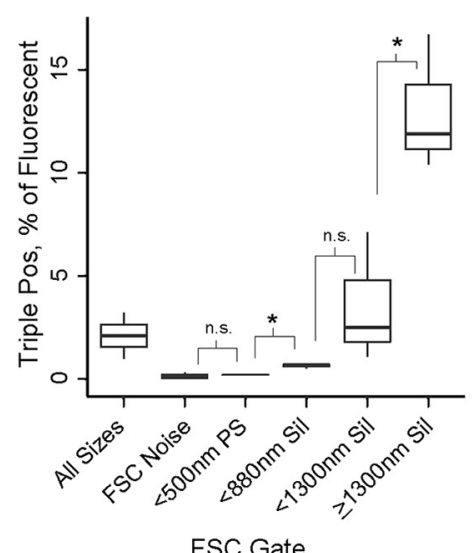

Double-Positive

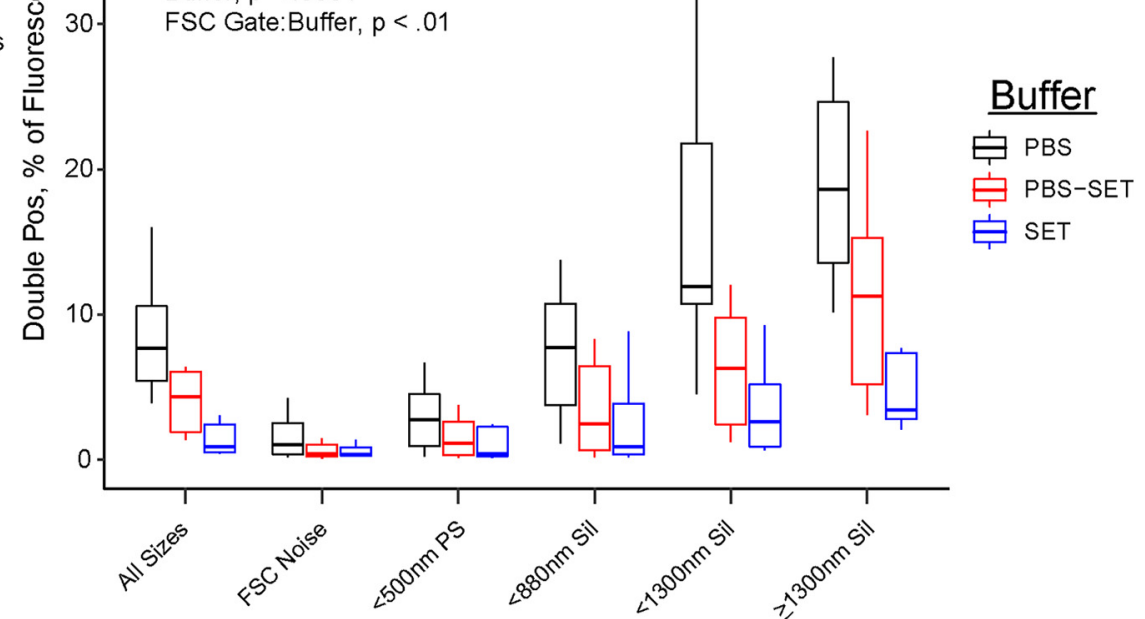

FSC Gate

Figure 6. Distribution of double-positive P2 events across FSC ranges and in different sample buffers. These experiments were 


\section{continued}

conducted using FM triggering on the Influx. $\boldsymbol{A}$, Representative density plot of non-fluorescent silica and fluorescent PS beads (Apogee) showing the five FSC-gated regions based on noise threshold and bead positions. $\boldsymbol{B}$, Same sample and FSC gates as in $\boldsymbol{A}$ but plotted to display green fluorescence of PS beads (FITC detector). $\boldsymbol{C}$, Representative density plot showing the five FSC-gated regions on a mixture of single calcein-labeled P2 samples. Only gated double-positive events are displayed. The percentage of all fluorescent (i.e., calcein-labeled) events that falls into each FSC gate is displayed in black (all five FSC gates sum to 100\%). The percentage of all calcein double-positive events that falls into each FSC gate is displayed in red (all five FSC gates sum to 100\%). The number of double-positive events within each FSC gate, expressed as a percentage of all fluorescent events in that FSC gate, is displayed in blue. $\boldsymbol{D}-\boldsymbol{F}$, Box and whiskers plots of single-positive, double-positive, or triple-positive events, expressed as a percentage of all fluorescent events within the respective FSC gate. Central bar represents the median. Lower and upper edges correspond to 25th and 75th percentiles. Lower and upper whiskers extend to the smallest or largest value no greater than 1.5 times the interquartile range away from the corresponding edge. Data for single-positive and double-positive events are derived from two-color mixtures of P2 samples single-labeled with calcein AM/calcein red $(n=4)$, violet amine-reactive dye/MitoTracker Deep Red FM $(n=1)$ or calcein AM $(n=1)$ or calcein red $(n=1)$, as well as three-color mixtures single-labeled with violet amine-reactive/calcein AM/calcein red $(n=3)$. Triple-positive event data are derived only from the three-color mixtures. A Student's $t$ test was performed comparing each FSC gate to that directly above or below it; $* p<0.05, * * p<0.01$. G, Diagram of buffer scheme during sample preparation. PBS and SET samples are labeled, washed, and run on the flow cytometer in their respective buffers. PBS-SET samples undergo calcein labeling and the first two washes in PBS before mixing, a third wash, and flow cytometry in SET buffer. $\boldsymbol{H}$, Box and whiskers plot of double-positive events, expressed as a percentage of all fluorescent events within the respective FSC gate. Data are derived from two-color mixtures of P2 samples single-labeled with calcein AM/calcein red using the indicated buffer scheme: PBS $(n=10)$, PBS-SET $(n=8)$, or SET $(n=10)$. Two-way ANOVA was performed, revealing significant main effects of FSC gate (Df $=$ $5, F=20.06, p=2.7 \mathrm{e}-15)$ and buffer $(\mathrm{Df}=2, F=20.14, p=1.8 \mathrm{e}-8)$, as well as a significant interaction $(\mathrm{Df}=10, F=2.78, p=0.004)$. See also Extended Data Figure 6-1.

VMAT2+NGAT+). We wondered whether the frequency of these double-positive subtypes was related to the overall abundance of their respective antigens (Extended Data Fig. 7-1C). We used the percentage of all events immunopositive for each antigen to construct a simple probability model. In this model, a double-positive event represents the random sampling of two events with replacement. The probability of a particular double-positive subtype is therefore obtained by multiplying the frequencies of its respective event types (Extended Data Fig. 7-1D). Consistent with the expected frequencies of double-positive subtypes, we found that VGLUT1+NGAT+ events were by far the most abundant ( $\sim 75 \%$ ), followed by VGLUT1+NMAT2+ ( 20\%), and finally VMAT2+NGAT+ ( 5\%; Extended Data Fig. 7-1D). These results are consistent with a simple "collision" model of aggregation in the P2 crude synaptosome preparation. Furthermore, they strongly suggest that many doublepositive events observed in these immunostaining experiments are aggregated particles rather than single synaptosomes co-expressing multiple vesicular transporters.

Although scatter measurements of submicron particles may vary dramatically across cytometers, fluorescence measurements can be readily calibrated. We determined the fluorescence intensity of VGAT/anti-mouse Alexa Fluor 488 immunostaining in molecules of equivalent soluble fluorophore (MESF) using calibrated bead standards (Extended Data Fig. 7-1E). Because particle fluorescence is directly related to FSC intensity regardless of the immunostaining procedure, many immunopositive events at a given FSC intensity would exhibit fluorescence intensity less than or equal to immunonegative events at a higher FSC intensity nearby. We therefore analyzed the median fluorescence intensity of gated immunopositive and immunonegative events within each FSC gate (Extended Data Fig. 7-1F). Numerical MESF data are also included in
Extended Data Figure 7-3, which should facilitate comparison across laboratories.

\section{Particle recovery following repeated centrifugation}

Given the increased frequency of aggregates after centrifugation (Fig. 5D), in higher FSC regions (Fig. $6 D-F$ ), and in PBS (Fig. 6H), we wondered whether buffer and centrifugation might interact to bias sample composition toward aggregates following repeated centrifugations. Indeed, Gray and Whittaker (1962) previously reported increased recovery of acetylcholine in the low-speed P1 pellet when using saline instead of sucrose buffer, which was interpreted as aggregation in saline increasing the effective size of synaptosomes (and thus sedimentation at lower speed). To assess whether multiple centrifugation steps might bias particle recovery in PBS versus SET, we conducted two sequential 5-min centrifugations of P2 samples in PBS or SET buffer at varying speeds and used flow cytometry with absolute counting beads to determine the particle concentration of the pellets and supernatants (Fig. 8A). We found that under carefully controlled conditions with the absolute counting beads, PBS reduced particle abundance by $\sim 3$-fold before any centrifugation (Fig. 8B). It is possible that certain particles in the P2 sample are susceptible to destruction in PBS, but several features of the data suggest that aggregation contributes to the reduction in particle counts. First, the median fluorescence intensity of membrane staining by FM4-64 was higher in PBS (Fig. 8B), which was due to increased relative abundance of brighter, larger events with higher FSC (Fig. $8 C, D$ ). In line with the findings of Gray and Whittaker (1962), we found that these "FSC high" particles were more efficiently pelleted in PBS at lower relative centrifugal force $(2500-5000 \times g)$ compared to SET buffer (Fig. 8D). The relative abundance of these particles was dramatically increased in the two pellets, P2-1 and P2-2, compared to the input sample and two superna- 
A
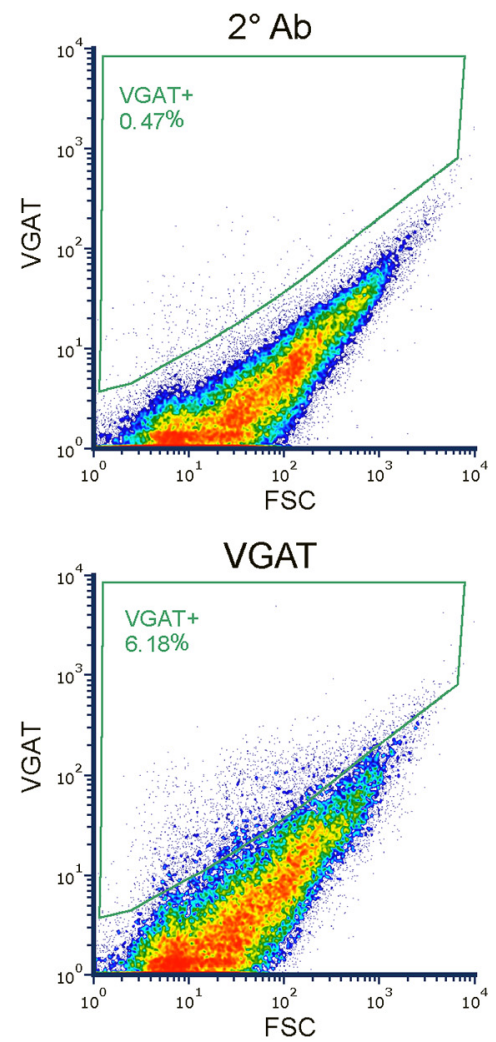

B

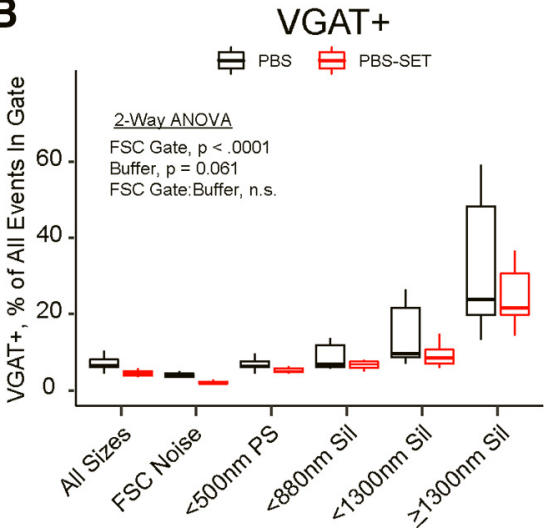

FSC Gate
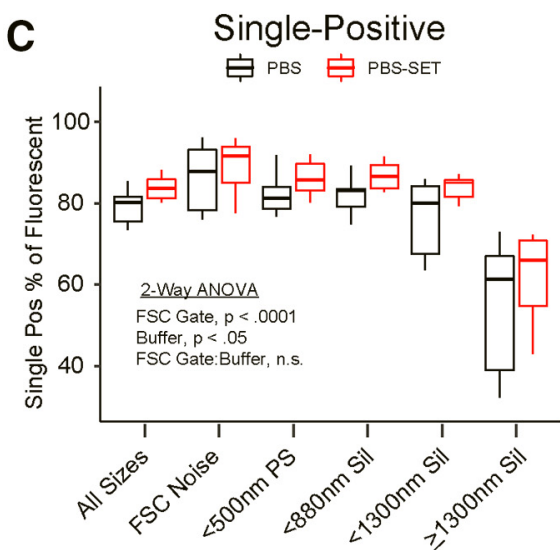

FSC Gate
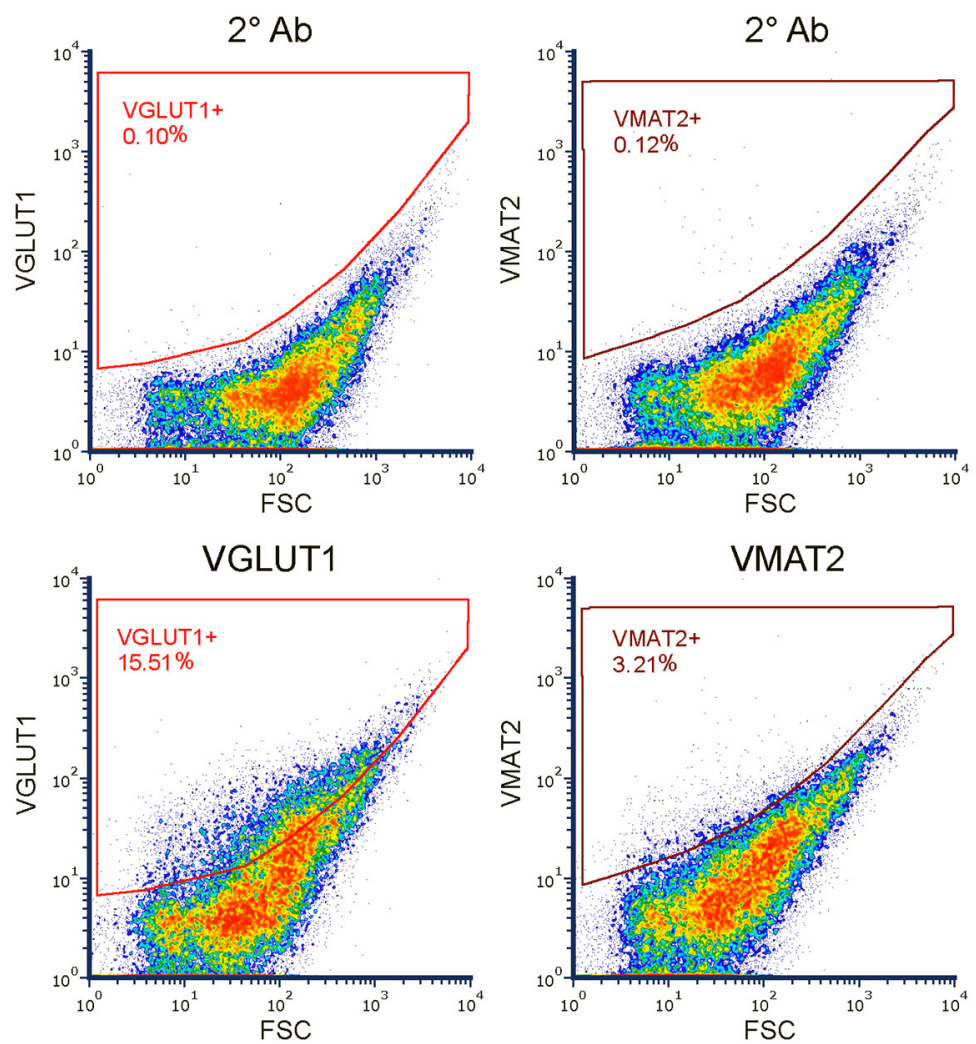

VGLUT1+

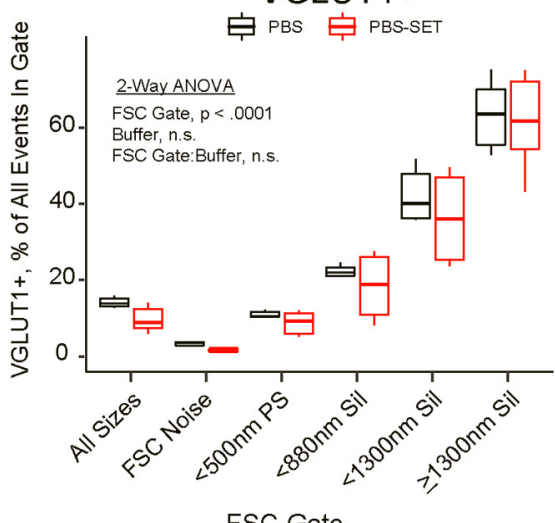

FSC Gate

\section{Double-Positive \\ 追 PBS 追 PBS-SET}

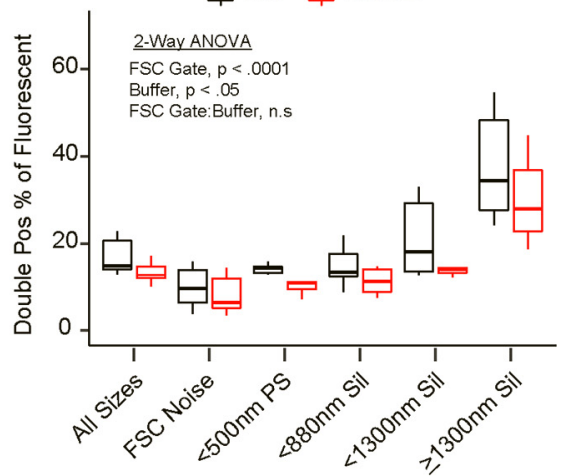

FSC Gate
VMAT2+

官 PBS 官 PBS-SET

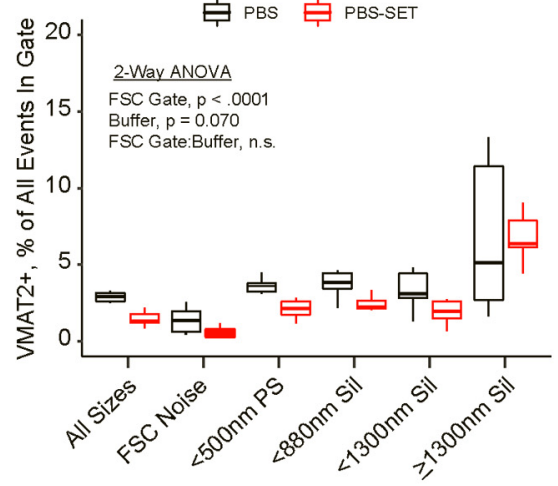

FSC Gate

Triple-Positive

追 PBS 追 PBS-SET

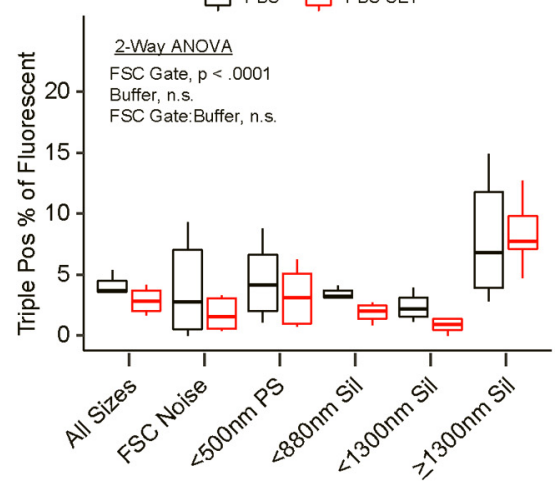

FSC Gate

Figure 7. Fractional abundance of immunostained P2 events in different FSC ranges and in different sample buffers. These 


\section{continued}

experiments were conducted using FM triggering on the Influx. $\boldsymbol{A}$, Representative density plots of P2 samples immunostained for VGAT, VGLUT1, and VMAT2 and run in PBS. Top, Secondary-only controls in which primary antibodies were omitted. Bottom, Immunostained with indicated primary antibodies. B, Box and whiskers plot of immunostained events as indicated, expressed as a percentage of all events within the respective FSC gate. Central bar represents the median. Lower and upper edges correspond to 25th and 75th percentiles. Lower and upper whiskers extend to the smallest or largest value no greater than 1.5 times the interquartile range away from the corresponding edge. Data are derived from P2 samples triple stained and washed in PBS, and subsequently run in either PBS $(n=6)$ or SET $(n=6)$ as sample buffer. Two-way ANOVA was performed for each dataset. Left, For VGAT+ events, FSC gate $(\mathrm{Df}=5, F=21.51, p=2.9 \mathrm{e}-12)$ and buffer $(\mathrm{Df}=1, F=4.70, p=0.034)$ were both significant, with no significant interaction (Df $=5, F=0.40, p=0.85$ ). Middle, For VGLUT1 + events, only FSC gate (Df $=5, F=68.9, p<2 e-16$ ) was significant, while buffer (Df $=1, F=0.36, p=0.55)$ and the interaction ( $\mathrm{Df}=5, F=0.19, p=0.97)$ were not. Right, For VMAT2 + events, FSC gate (Df $=$ $5, F=12.54, p=2.4 \mathrm{e}-8$ ) and buffer ( $\mathrm{Df}=1, F=5.21, p=0.026)$ were both significant, with no significant interaction (Df $=5, F=$ $0.27, p=0.93$ ). $C$, Box and whiskers plots of single-positive, double-positive, and triple-positive events expressed as a percentage of all fluorescent events within the respective FSC gate. Data are derived from the same immunostained P2 samples as in $\boldsymbol{B}$. Two-way ANOVA was performed for each dataset. Left, For single-positive events, FSC gate (Df $=5, F=19.83$, $p=1.3 e-11)$ and buffer (Df $=1, F=6.74, p=0.012$ ) were both significant, with no significant interaction ( $\mathrm{Df}=5, F=0.15, p=0.98)$. Middle, For double-positive events, FSC gate ( $\mathrm{Df}=5, F=23.25, p=6.6 \mathrm{e}-13$ ) and buffer ( $\mathrm{Df}=1, F=8.00, p=0.006$ ) were both significant, with no significant interaction ( $\mathrm{Df}=5, F=0.40, p=0.85)$. Right, For triple-positive events, only FSC gate $(\mathrm{Df}=5, F=8.37, p=4.7 \mathrm{e}-6)$ was significant, while buffer ( $\mathrm{Df}=1, F=1.91, p=0.17$ ) and the interaction ( $\mathrm{Df}=5, F=0.19, p=0.97$ ) were not. See also Extended Data Figures 7-1, 7-2, 7-3.

tants (Fig. 8E). The relative abundance of the large particles in PBS samples was highest in $2500 \times g$ pellets and lowest in $10,000 \times g$ pellets, while no clear relationship between relative abundance and centrifugation speed was observed in SET buffer (Fig. 8F). A slight increase in these FSC high particles was observed when comparing spin 1 versus spin 2 for both buffers, but this effect was modest compared to the increase in PBS versus SET (Fig. $8 F$ ).

As expected, the overall efficiency of particle recovery in the pellet of each spin increased with centrifugation speed, with the exception of the first spin in PBS (Extended Data Fig. 8-1A-C). Similar to the input samples, it appears that a combination of aggregation and/or destruction contribute to the loss of particle counts in the first PBS spin, although the recovery in the second spin was higher than in SET buffer. Critically, the first 5-min spin at 2500-5000 $\times g$ in either buffer leaves over half the P2 particles in the supernatant (Extended Data Fig. 8-1D). Collectively, these data suggest that P2 samples do undergo a certain degree of further fractionation during repeated centrifugation-based wash steps. Although some centrifugation bias is inevitable regardless of buffer and centrifugation speed, our data suggest that lower centrifugation speeds and ionic buffers will bias the overall sample composition toward larger particles that are more likely to be aggregates.

\section{Discussion}

The goal of most flow cytometry experiments is to accurately detect and quantify the fluorescence intensity of single particles or cells in suspension. Toward that goal, we employed a variety of experimental assays that can be used to optimize synaptosome flow cytometry experiments. We have summarized our approach in Table 5 and will mirror the workflow in our discussion. We note that this workflow could be employed for any type of small particle, but our discussion will focus on aspects specific to synaptosomes.

\section{FSC triggering and bead-based size estimation}

It is now widely accepted that FSC-based size estimation using PS beads underestimates biological particle size (Nolan, 2015; Lannigan et al., 2016). This consequence of the refractive index mismatch has been experimentally validated in numerous flow cytometry studies (Chandler et al., 2011; van der Pol et al., 2012; Simonsen, 2016). The extent of underestimation depends on a variety of factors including the scattering parameters used, refractive indices of the sample and beads, and optical configuration of the cytometer. Estimates of the lipid vesicle size range defined by PS beads have been conducted on various cytometers and are shown in Table 6.

Synaptosomes are heterogeneous in size, but a generally accepted size range is $0.5-1.0 \mu \mathrm{m}$ (Gray and Whittaker, 1962; Hollingsworth et al., 1985; Dunkley et al., 1986, 1988; Williams et al., 2009; Fernández-Busnadiego et al., 2013). Although certain preparations contain larger synaptosomes, such as Dunkley's Percoll fraction 4 from striatum (Robinson et al., 1989), most studies of cortical synaptosomes consistently obtain mean diameters of 2500-600 nm (Table 7). Based on the synaptosome size ranges in Table 7 and vesicle size ranges defined by PS beads in Table 6, a majority of single synaptosomes would be expected to produce lower FSC signals than 500-nm PS beads and therefore would be undetectable by FSC triggering on many cytometers. Indeed, we could detect neither 585-nm silica beads nor more than half of P2 samples by FSC triggering on the Fortessa (Fig. 1C,D). Although the greater sensitivity afforded by the Influx enabled detection of $585-\mathrm{nm}$ silica beads above the FSC noise threshold, more than half of the P2 sample was still undetectable by FSC triggering (Fig. 1C,D). Our results are consistent with those of Biesemann et al. (2014), who sorted fluorescent VGLUT1 synaptosomes from below the FSC noise threshold using FM triggering on a BD FACS Aria. These electron microscopy, immunostaining, and proteomic studies convincingly demonstrated that single synaptosomes can be purified by sorting events 


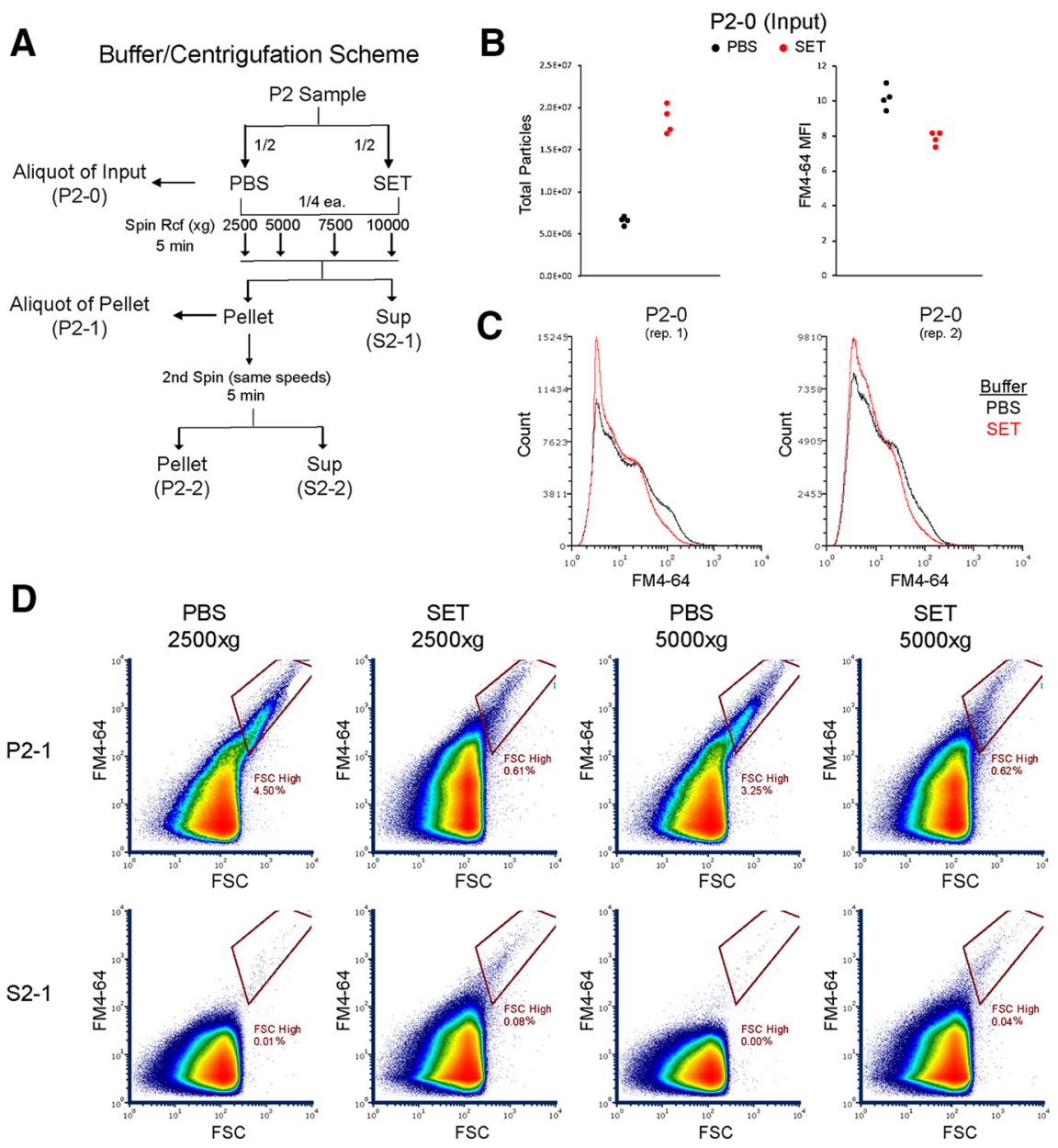

$\mathbf{E}$

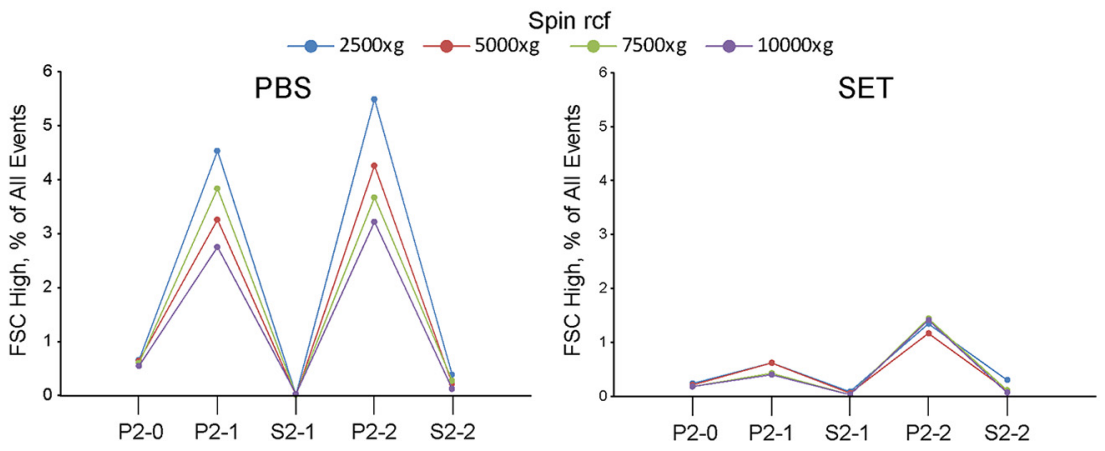

$\mathbf{F}$

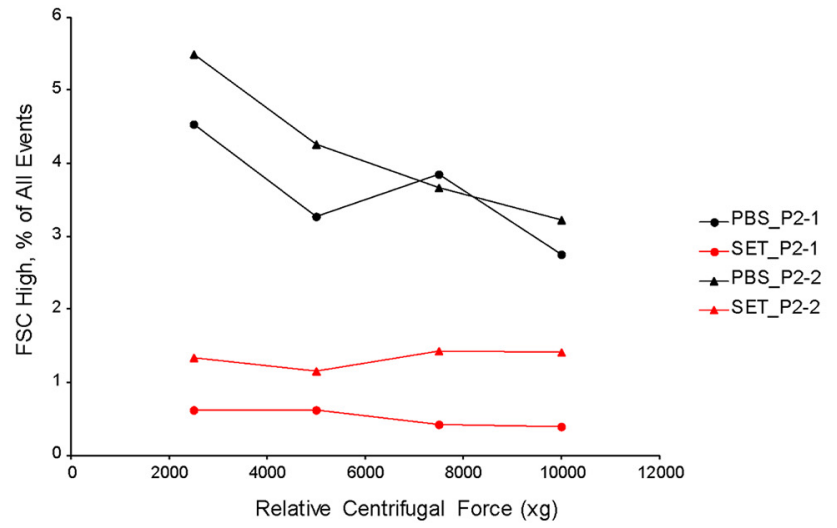

Figure 8. Effects of repeated centrifugation in different sample buffer on particle abundance and composition of P2 samples. These 


\section{continued}

experiments were conducted using FM triggering on the Influx. A, Diagram of buffer/centrifugation scheme. A P2 sample resuspended in PBS or SET was aliquoted to measure input (P2-0), followed by two centrifugations for 5 min at the indicated relative centrifugal force $(\times g)$. An aliquot of the first pellet (P2-1) is reserved before the second spin. All samples are then analyzed by flow cytometry using absolute counting beads to measure particle number in each fraction. $\boldsymbol{B}$, Input samples (P2-0) in PBS or SET buffer ( $n=4$ each). Left, Total particle counts. Right, Median fluorescence intensity of FM4-64 for all events. C, Representative histogram of FM4-64 fluorescence of all events for two replicate input samples (P2-0) in PBS and SET buffer. D, FSC versus FM4-64 density plots for P2-1 (top) or S2-1 (bottom) samples in PBS or SET buffer centrifuged at $2500 \times g$ (left) or $5000 \times g$ (right). FSC high gate corresponds to the top two FSC gates from Figures 6, 7. E, Gated FSC high events, expressed as \% of all events, plotted for PBS (left) or SET (right) in each of five fractions moving through the centrifugation protocol at indicated relative centrifugal force $(\times g)$. Left to right in each panel, Input (P2-0), first pellet (P2-1) and supernatant (S2-1), second pellet (P2-2) and supernatant (S2-2). $\boldsymbol{F}$, Gated FSC high events, expressed as \% of all events, for P2-1 and P2-2 samples in PBS or SET at indicated relative centrifugal force $(\times g)$. See also Extended Data Figure 8-1.

that fall below the FSC noise threshold, which was well below 750-nm PS beads on their cytometer (Biesemann, 2010; his Results 3.5, Fig. 15, pp 87-90).

In contrast, another widely adopted protocol (Gylys and Bilousova, 2017) implements FSC triggering and specifically analyzes only events in the FSC range defined by 0.5 - to 1.5- $\mu \mathrm{m}$ PS beads. Based on the high percentage of events that carry immunofluorescent signal for synaptic markers, these events are assumed to represent single synaptosomes and material below $500-\mathrm{nm}$ PS beads is regarded as debris (Gylys et al., 2004). One study analyzed "large synaptosomes," events in an FSC range defined by 1.5- to 4.5- $\mu \mathrm{m}$ PS beads (Gylys et al., 2000). We found that the FSC range above $2-\mu \mathrm{m}$ PS beads includes particles as large as neuronal nuclei (Extended Data Fig. 1-1C). To our knowledge, the only single synaptosomes approaching this size are derived from mossy fiber terminals of the hippocampus and cerebellum, and accordingly these synaptosomes sediment along with nuclei in the P1 fraction (Taupin et al., 1994; Terrian et al., 1988). In our

Table 5. Summary of key steps in microparticle flow cytometry experimental workflow

\begin{tabular}{lll}
\hline \multicolumn{1}{c}{ Purpose } & \multicolumn{1}{c}{ Relevant figures } & \multicolumn{1}{c}{ Experimental assays } \\
$\begin{array}{l}\text { Determine detection sensitivity } \\
\text { of cytometer }\end{array}$ & Fig. 1; & FSC vs fluorescence triggering with \\
Extended Data Fig. 1-1 & $\begin{array}{l}\text { lipid dye (e.g., FM4-64 or other) } \\
\text { PS and silica bead standards }\end{array}$
\end{tabular}

Determine linear range of particle detection

Detection of coincidence and aggregation

Distinguishing between coincidence and aggregation

Reduce aggregation

dentify suitable FSC ranges

Assess false colocalization of antigens
Fig. 2;

Extended Data Fig. 2-1

Fig. 3;

Extended Data Fig. 3-1

Fig. 4;

Extended Data Fig. 4-1 Fig. 5

Dilution series with multicolor

(2) Sorting double-positive events

Fig. 6;

Extended Data Fig. 6-1 Fig. 7;

Extended Data Fig. 7-1

Fig. 6;

Extended Data Fig. 6-1 Fig. 7;

Extended Data Fig. 7-1

Fig. 7;

Extended Data Fig. 7-1 dye labeling from beads vs samples (3) Spin vs mix multicolor

dye labeling

Dilution series

Multicolor dye labeling

Use of nonionic buffers

Immunostaining and multicolor dye labeling

Immunostaining of mutually exclusive antigens
Key notes

A number of dyes are suitable for

fluorescence triggering (Gray et al., 2015

Arraud et al., 2016; Kormelink et al., 2016;

Stoner et al., 2016). Multiple studies,

including ours, have demonstrated the

increased sensitivity, accuracy,

and reliability of fluorescence

triggering (Nolan and Stoner, 2013;

Arraud et al., 2016).

FSC signal from bead standards

(especially PS) does NOT accurately

reflect the size of

biological particles detected.

Refractive index differences lead to size

underestimation, the extent of which

is highly cytometer dependent (Table 6).

Conducting a dilution series is critical

to identify the range of linear

detection on each cytometer.

A number of dyes are suitable for

multicolor dye labeling assays,

as described in cellular "barcoding"

assays (Krutzik and Nolan, 2006;

Krutzik et al., 2011).

We found that mixtures of different

color PS beads are a suitable

model of coincidence. Double-positive

sample events that do not decrease

with dilution, and those that

can be enriched by sorting, strongly

suggest aggregates vs coincidence.

This can be further supported

by testing whether the frequency

of double-positive events is increased

by centrifugation.

As reported in previous microscopy

studies (Choi et al., 2009; Daniel et al., 2012), we

found that nonionic sample buffers

tend to reduce aggregation of synaptosomes.

This is not a complete solution

to eliminating false double-positive

and triple-positive events.

FSC of beads provides relative references

for avoiding regions with high

false double-positive rates

Highly abundant antigens that should

be mostly exclusive are best

for these experiments. 
Table 6. Previous comparisons of PS bead versus lipid vesicle scattering measurements

\begin{tabular}{|c|c|c|c|c|}
\hline Study & Cytometer model & Parameter & PS bead range & Vesicle range \\
\hline Chandler et al. (2011) & Apogee A40 & FSC & $0.5-1.0 \mu \mathrm{m}$ & $1.4-2.7 \mu \mathrm{m}$ \\
\hline van der Pol et al. (2012) & Beckman Coulter FC 500 & FSC & $0.5-0.9 \mu \mathrm{m}$ & $1.00-1.75 \mu \mathrm{m}$ \\
\hline van der Pol et al. (2012) & Apogee A40 & FSC & $0.5-0.9 \mu \mathrm{m}$ & $1.25-2.0 \mu \mathrm{m}$ \\
\hline van der Pol et al. (2012) & Becton Dickinson FACSCalibur & SSC & $0.5-0.9 \mu \mathrm{m}$ & $2.3-4.6 \mu \mathrm{m}$ \\
\hline Simonsen (2016) & $\begin{array}{l}\text { Becton Dickinson } \\
\text { LSRII }\end{array}$ & SSC & $110 \mathrm{~nm}$ & $400 \mathrm{~nm}$ \\
\hline
\end{tabular}

hands, the FSC region above 1.3- $\mu \mathrm{m}$ silica beads (which would fall below both 1.5- and 4.5- $\mu \mathrm{m}$ PS beads) represents $<5 \%$ of all events in the P2 sample (Fig. $6 C$ ) and contains the highest frequency of false double-positive events (Figs. $6 E-H, 7 C$ ). Our results strongly suggest that a large fraction of events in these regions are synaptosome-containing aggregates, while single synaptosomes are often found below 500-nm PS beads and the FSC noise threshold.

\section{Fluorescence triggering and dilution to control for coincidence}

Maintaining an appropriately low sample concentration is critical to prevent coincident particle detection in submicron flow cytometry experiments. It has been suggested that coincidence is especially prominent when operating in FSC-trigger mode (Nolan and Stoner, 2013), which might explain the variations in FSC and FM4-64 fluorescence we observed with dilution in FSC-trigger mode on both cytometers (Fig. 2C,D). Importantly, we found that FSC-trigger mode substantially underestimates the true event rate in P2 samples on both cytometers. In such a situation, undetected particles in the sample volume associated with a detectable particle can contribute to the fluorescence measurements and/or be sorted in the same drop (Libregts et al., 2018). Thus, with FSC triggering at high event rates, one cannot determine if sorted material accurately reflects the detection and sorting of single particles. Although we found that aggregation was the major source of double-positive events in our samples, we did not conduct these assays using FSC-trigger mode or at high event rates. Serial dilutions and fluorescence triggering should be conducted in all submicron flow cytometry experiments to avoid coincidence.

\section{Detection of coincidence and aggregation}

To our knowledge, our study is the first to systematically address coincidence and aggregation in flow cytometric analysis of synaptosomes (Fig. 3). We chose a strategy analogous to a "mixed-species" experiment commonly employed to validate single cell RNAsequencing systems, where a mixture of mouse and human cells is analyzed to quantify cell-doublet rates (Klein et al., 2015; Macosko et al., 2015). Our dye labeling strategy is also employed in high-throughput flow cytometry studies, where cells from different conditions are given non-transferable fluorescent barcodes before mixing, staining, and acquisition in a single batch (Krutzik et al., 2011). Although the cellular fluorescent barcodes are decoded with high accuracy in these protocols, even when multiple barcodes are encoded by discrete concentrations of a single dye (Krutzik and Nolan, 2006), our results demonstrate that synaptosome preparations do not behave similarly to cells in these experiments. Determining the extent of single-particle detection in submicron flow cytometry experiments is a challenging task. Image cytometry, which combines confocal microscopy with the fluidics of a cytometer, is powerful in this regard since images of each event can be manually examined after

Table 7. Previous size range measurements of mammalian synaptosomes

\begin{tabular}{|c|c|c|c|c|}
\hline Study & Method & Species/region & Synaptosome fraction & Mean diameter ( \pm SEM) \\
\hline Dunkley et al. (1986) & Electron microscopy & Rat/cortex & P2 Percoll fraction 3 & $515 \pm 17 \mathrm{~nm}$ \\
\hline Dunkley et al. (1986) & Electron microscopy & Rat/cortex & P2 Percoll fraction 4 & $568 \pm 14 \mathrm{~nm}$ \\
\hline Dunkley et al. (1986) & Electron microscopy & Rat/cortex & P2 Percoll fraction 5 & $539 \pm 12 \mathrm{~nm}$ \\
\hline Dunkley et al. (1988) & Electron microscopy & Rat/cortex & S1 Percoll fraction 1 & $320 \pm 130 \mathrm{~nm}$ \\
\hline Dunkley et al. (1988) & Electron microscopy & Rat/cortex & S1 Percoll fraction 2 & $460 \pm 150 \mathrm{~nm}$ \\
\hline Dunkley et al. (1988) & Electron microscopy & Rat/cortex & S1 Percoll fraction 3 & $550 \pm 130 \mathrm{~nm}$ \\
\hline Dunkley et al. (1988) & Electron microscopy & Rat/cortex & S1 Percoll fraction 4 & $640 \pm 120 \mathrm{~nm}$ \\
\hline Dunkley et al. (1988) & Electron microscopy & Rat/cortex & S1 Percoll fraction 5 & $630 \pm 190 \mathrm{~nm}$ \\
\hline Robinson et al. (1989) & Electron microscopy & Rat/striatum & S1 Percoll fraction 3 & $591 \pm 9 \mathrm{~nm}$ \\
\hline Robinson et al. (1989) & Electron microscopy & Rat/striatum & S1 Percoll fraction 4 & $905 \pm 12 \mathrm{~nm}$ \\
\hline Hollingsworth et al. (1985) & Electron microscopy & Guinea pig/cortex & $\begin{array}{l}\text { Filtration } \\
\text { synaptoneurosomes }\end{array}$ & $560 \pm 15 \mathrm{~nm}$ \\
\hline Hollingsworth et al. (1985) & Light microscopy & Guinea pig/cortex & $\begin{array}{l}\text { Filtration } \\
\text { synaptoneurosomes }\end{array}$ & $\sim 600 \mathrm{~nm}$ \\
\hline Williams et al. (2009) & Phase contrast microscopy & Human/cortex & $\begin{array}{l}\text { Filtration } \\
\text { synaptoneurosomes }\end{array}$ & $300 \pm 700 \mathrm{~nm}$ \\
\hline $\begin{array}{l}\text { Fernández-Busnadiego } \\
\text { et al. (2013) }\end{array}$ & Cryo-electron tomography & Mouse/cortex & Percoll fraction $3+4$ & $500 \pm 1000 \mathrm{~nm}$ \\
\hline
\end{tabular}


acquisition. However, these instruments require extensive optimization and are not widely available to all labs. The fluorescent labeling and mixing assay we describe here can be implemented on most conventional flow cytometers and should be broadly useful to the microparticle flow cytometry community.

\section{Distinguishing between coincidence and aggregation}

As we were unable to reduce the frequency of false double-positive events with extensive dilution and FM triggering of our P2 mixtures (Fig. 4C,D), we began to suspect aggregation as the source of these events. Aggregated particles would behave as "single" particles in terms of dilution and coincidence, but nonetheless present serious problems for both cellular and submicron flow cytometry analysis. The large scatter signals produced by cells result in abnormal width/height versus area pulse profiles, which can be used to remove cell-doublet events in conventional flow cytometry experiments. To date, we are unaware of any study demonstrating that this gating strategy works on submicron particles, and our results strongly suggest it does not (Extended Data Fig. 3-1C). We further demonstrated that double-positive events in our P2 mixtures can be enriched by sorting (Fig. $5 A-C$ ), while fluorescent PS bead double-positive events are depleted by sorting. These results are highly consistent with our dilution series and provide further evidence that the dominant source of double-positive events in P2 mixtures is aggregation. Combined with our fluorescent labeling and mixing assay, comparison to fluorescent PS bead mixtures in dilution series and sorting experiments provides a general strategy to determine the extent of coincidence versus aggregation in any sample type.

\section{Synaptosome aggregation}

Single events comprised of aggregated cell-derived microparticles have been definitively identified using image cytometry (Erdbrügger et al., 2014). It is unclear how often and under what conditions cell-derived microparticles aggregate, but one can speculate that their endogenous function within physiologic bodily fluids would make them somewhat resistant to aggregation. In contrast to cellderived microparticles, presynaptic nerve terminals do not exist as soluble particles in vivo, and the aggregation of synaptosomes in ionic media (saline-based solutions such as PBS) is a well-described phenomenon. Gray and Whittaker (1962) analyzed the minimum concentration of various electrolytes required to induce aggregation of particles in the P2 and sucrose-gradient synaptosome fractions. They describe how the particles behave as though negatively charged, since divalent and trivalent cations are particularly effective in causing aggregation. However, the minimum concentration for sodium chloride was $20 \mathrm{mM}$ (Gray and Whittaker, 1962), far below the 137 $\mathrm{mM}$ present in PBS. Others encountered such aggregates using a filtration procedure to purify "synaptoneurosomes" from brains homogenized in saline (Hollingsworth et al., 1985).

Most synaptosome flow cytometry studies have employed ionic media for incubation, washing, and sample analysis, while assuming that each event generated by the flow cytometer represents a single synaptosome (Gylys et al., 2000, 2004; Fein et al., 2008; Postupna et al., 2014; Prieto et al., 2017). Studies of cell-derived microparticles have clearly demonstrated situations where this assumption breaks down (Erdbrügger et al., 2014; Libregts et al., 2018), and this issue was recently brought forth in synaptosome flow cytometry (see comments on Prieto et al., 2017). The centrifugation and time-lapse variations of our fluorescent mixture experiments provide further insight into particle aggregation in P2 samples (Fig. 5D). Most strikingly, we found that centrifugation and resuspension of the single-labeled mixture dramatically increases the frequency of double-positive events. This finding strongly suggests that resuspension of the P2 pellet does not completely eliminate particles that aggregate during centrifugation. We also observed a steady increase in the frequency of double-positive events over time with or without centrifugation (Fig. 5D), suggesting that aggregation is an ongoing process in P2 sample mixtures. These results also further argue against coincidence as a cause of these double-positive events, as coincidence would not be expected to increase over time. Although washing steps are critical to these experiments, our data suggest that repeated centrifugation will bias the sample composition. The enhanced relative abundance of larger, brighter particles following centrifugation in PBS (Fig. $8 D-F$ ), especially at low speeds, is consistent with previous work (Gray and Whittaker, 1962) and provides further evidence that many of these high FSC events are aggregated particles. Future studies should carefully consider the effects of duration, speed, and number of centrifugations on downstream experimental results.

\section{Reducing synaptosome aggregation}

Analogous to the microscopy results of other groups (Choi et al., 2009; Daniel et al., 2012), we found that SET buffer significantly reduced the frequency of doublepositive events in our P2 mixtures, especially in the higher FSC gates (Fig. 6H). However, calcein-labeling efficiency was also lower in SET buffer (Extended Data Fig. 6-1C) and SET would not be compatible with many physiologic assays (Choi et al., 2009). We found that conducting the labeling procedure in PBS and switching to SET buffer for flow cytometry also reduced the double-positive event frequency, although the effect was smaller than for SET alone (Fig. 6H). Our flow cytometry assay provides a quantitative measure (albeit an underestimate) of synaptosomal aggregation in each buffer condition and should be useful to monitor the impact of future protocol improvements. Even without the fluorescent mixture assay, we observed that SET buffer reduces the relative abundance of particles in the highest FSC regions most likely to contain aggregates (Fig. 8D-F). Anecdotally, we noticed that the P2 pellet disperses relatively easily in SET buffer compared to PBS. Based on the findings of Gray and Whittaker (1962), we suspect that removing cations reduces aggregation by allowing the negatively charged neuronal membranes to repel each other. 


\section{Pitfalls in immunophenotyping of synaptosomes}

The heterogeneity of presynaptic nerve terminals, including specific cases of neurotransmitter co-release (Hnasko and Edwards, 2012; Vaaga et al., 2014), makes high-throughput immunophenotyping of synaptosomes by neurotransmitter content an appealing direction for synaptosome flow cytometry. Indeed, Gajera et al. (2019) have recently demonstrated high-dimensional analysis of human synaptosomal preparations using mass cytometry. Our results suggest that the presence of synaptosomal aggregates is a serious problem for such experiments, especially in the top two FSC ranges in our study (higher than 880-nm silica beads; Fig. 7C). We found implausibly high co-labeling of VGAT, VGLUT1, and VMAT2 in our P2 samples (Extended Data Fig. 7-2), and the increasing frequency of double-positive and triplepositive events observed across FSC gates corroborates the analogous finding in our fluorescent mixture assay (Figs. $6 D-F, 7 C$ ). These findings further support our conclusion that a high percentage of events in this FSC range are synaptosome-containing aggregates. As expected based on synapse abundance, VGAT+/VGLUT1+ double-positive events were observed most frequently (Extended Data Fig. 7-1C,D). Similarly, Biesemann previously demonstrated co-enrichment of VGAT and VGLUT1 by sorting VGLUT1 fluorescent events between 0.75- and 1.5- $\mu \mathrm{m}$ beads (Biesemann, 2010; his Results 3.4, Fig. 14, pp 86-87). Western blotting of the sorted material revealed enrichment of VGAT and myelin proteolipid protein (PLP), proteins that should be depleted in a sort of pure VGLUT1 + synaptosomes. After implementing FM triggering and sorting only VGLUT1 fluorescent events below the FSC noise threshold, the authors demonstrated unprecedented purity of sorted VGLUT1 synaptosomes with the expected depletion of VGAT and other glial markers (Biesemann et al., 2014). Overall, our results are highly consistent with their conclusion that the previously sorted events were aggregates containing at least one glutamatergic synaptosome (Biesemann, 2010; his Results 3.4, p 86).

As others have pointed out (Erdbrügger et al., 2014), immunophenotyping of submicron particles can result in detection, but not necessarily accurate quantification. Unfortunately, we found that SET buffer was less effective in reducing false double-positive and triple-positive immunostained events (Fig. 7C) compared to those observed in the fluorescent mixture assay (Fig. 6H). Since resuspension of the P2 pellet is unlikely to completely eliminate aggregates, we suspect that some aggregated particles are cross-linked during formaldehyde fixation before immunostaining. Accordingly, we found that single-positive event frequency was generally $\sim 90 \%$ below the FSC noise threshold in immunostained samples (Fig. 7C), while the single-positive frequency in this region was generally $\sim 97 \%$ in the calcein mixtures (Fig. 6D). Although this suggests that some of the double-positive events in the immunostained samples are single synaptosomes with true colocalization (e.g., VGAT+NGLUT1+ nerve terminals in cortical Layer V; Fattorini et al., 2009), distinguishing such events from aggregates would require extensive experimental validation. Flow cytometry studies claiming to study single synaptosomes often report a high-degree of co-labeling for markers that are expected to colocalize, such as VGLUT1, PSD95, synaptophysin, SNAP25, etc. (Gylys et al., 2004; Prieto et al., 2017). Such data does not provide evidence that the detected particles are single synaptosomes, since synaptosome-containing aggregates would be expected to yield similar, if not even higher co-labeling for these markers. Instead, we propose that such studies should explicitly report the co-labeling of neurotransmitter-specific markers (i.e., VGLUT1, VGAT, etc.) that should be mutually exclusive for most single synaptosomes. As suggested previously (Nolan and Stoner, 2013), claims of co-localization should be supported by direct evidence that coincidence and aggregation are not contributing to the measurement.

\section{Challenges and future directions}

Here, we highlighted a number of technical challenges that hinder the detection and analysis of single particles in synaptosome flow cytometry. In the absence of new purification methods, synaptosome preparations will inevitably be contaminated with membranes, myelin, and mitochondria. Currently, it seems that transgenic mice harboring fluorescent protein reporters are required for sorting, since tractable surface markers are limited and most downstream assays are not compatible with fixation and immunostaining of cytoplasmic proteins. A sorting protocol for VGLUT1 Venus synaptosomes has described by Herzog and colleagues (Biesemann et al., 2014; Luquet et al., 2017; see also Poulopoulos et al., 2019 for growth cone sorting) and should be readily adaptable for other fluorescent proteins and synapse types. However, if immunostained synaptosome events are the analytical endpoint for an experiment, the methods described in our study will minimize the effects of coincidence and aggregation on the analysis of single synaptosomes. Such procedures will need to be routinely performed on each cytometer, and confirmation of accuracy by microscopy seems prudent. We remain optimistic that future advances in instrumentation, reagents, and sample preparation will obviate the need for such extensive QC. Despite current limitations, we expect synaptosome flow cytometry studies to continue enhancing our understanding of synaptic biology in health and disease.

\section{References}

Arraud N, Gounou C, Turpin D, Brisson AR (2016) Fluorescence triggering: a general strategy for enumerating and phenotyping extracellular vesicles by flow cytometry. Cytometry A 89:184-195.

Biesemann C (2010) Development of fluorescence activated synaptosome sorting (FASS) and analysis of VGLUT1 synapses from mouse brain. Göttingen: Georg August University Göttingen.

Biesemann C, Grønborg M, Luquet E, Wichert SP, Bernard V, Bungers SR, Cooper B, Varoqueaux F, Li L, Byrne JA, Urlaub H, Jahn O, Brose N, Herzog E (2014) Proteomic screening of glutamatergic mouse brain synaptosomes isolated by fluorescence activated sorting. EMBO J 33:157-170.

Chandler WL, Yeung W, Tait JF (2011) A new microparticle size calibration standard for use in measuring smaller microparticles using a new flow cytometer. J Thromb Haemost 9:1216-1224.

Choi SW, Gerencser AA, Nicholls DG (2009) Bioenergetic analysis of isolated cerebrocortical nerve terminals on a microgram scale: spare respiratory capacity and stochastic mitochondrial failure. J Neurochem 109:1179-1191. 
Daniel JA, Malladi CS, Kettle E, McCluskey A, Robinson PJ (2012) Analysis of synaptic vesicle endocytosis in synaptosomes by highcontent screening. Nat Protoc 7:1439-1455.

Dunkley PR, Jarvie PE, Heath JW, Kidd GJ, Rostas JA (1986) A rapid method for isolation of synaptosomes on Percoll gradients. Brain Res 372:115-129.

Dunkley PR, Heath JW, Harrison SM, Jarvie PE, Glenfield PJ, Rostas JA (1988) A rapid Percoll gradient procedure for isolation of synaptosomes directly from an S1 fraction: homogeneity and morphology of subcellular fractions. Brain Res 441:59-71.

Erdbrügger U, Rudy CK, Etter ME, Dryden KA, Yeager M, Klibanov AL, Lannigan J (2014) Imaging flow cytometry elucidates limitations of microparticle analysis by conventional flow cytometry. Cytometry A 85:756-770.

Fattorini G, Verderio C, Melone M, Giovedì S, Benfenati F, Matteoli M, Conti F (2009) VGLUT1 and VGAT are sorted to the same population of synaptic vesicles in subsets of cortical axon terminals. J Neurochem 110:1538-1546.

Fein JA, Sokolow S, Miller CA, Vinters HV, Yang F, Cole GM, Gylys $\mathrm{KH}$ (2008) Co-localization of amyloid beta and tau pathology in Alzheimer's disease synaptosomes. Am J Pathol 172:1683-1692.

Fernández-Busnadiego R, Asano S, Oprisoreanu A-M, Sakata E, Doengi M, Kochovski Z, Zürner M, Stein V, Schoch S, Baumeister W, Luci V (2013) Cryo-electron tomography reveals a critical role of RIM1 $\alpha$ in synaptic vesicle tethering. J Cell Biol 201:725-740.

Gajera CR, Fernandez R, Postupna N, Montine KS, Fox EJ, Tebaykin D, Angelo M, Bendall SC, Keene CD, Montine TJ (2019) Mass synaptometry: high-dimensional multi parametric assay for single synapses. J Neurosci Methods 312:73-83.

Gleitz J, Beile A, Wilffert B, Tegtmeier F (1993) Cryopreservation of freshly isolated synaptosomes prepared from the cerebral cortex of rats. J Neurosci Methods 47:191-197.

Gray EG, Whittaker VP (1962) The isolation of nerve endings from brain. J Anat 96:79-88.

Gray WD, Mitchell AJ, Searles CD (2015) An accurate, precise method for general labeling of extracellular vesicles. MethodsX 2:360-367.

Gylys KH, Bilousova T (2017) Flow cytometry analysis and quantitative characterization of tau in synaptosomes from Alzheimer's disease brains. Methods Mol Biol 1523:273-284.

Gylys KH, Fein JA, Cole GM (2000) Quantitative characterization of crude synaptosomal fraction (P-2) components by flow cytometry. J Neurosci Res 61:186-192.

Gylys KH, Fein JA, Yang F, Cole GM (2004) Enrichment of presynaptic and postsynaptic markers by size-based gating analysis of synaptosome preparations from rat and human cortex. Cytometry A 60:90-96.

Hnasko TS, Edwards RH (2012) Neurotransmitter corelease: mechanism and physiological role. Annu Rev Physiol 74:225-243.

Hollingsworth EB, McNeal ET, Burton JL, Williams RJ, Daly JW, Creveling CR (1985) Biochemical characterization of a filtered synaptoneurosome preparation from guinea pig cerebral cortex: cyclic adenosine 3':5'-monophosphate-generating systems, receptors, and enzymes. J Neurosci 5:2240-2253.

Klein AM, Mazutis L, Akartuna I, Tallapragada N, Veres A, Li V, Peshkin L, Weitz DA, Kirschner MW (2015) Droplet barcoding for single cell transcriptomics applied to embryonic stem cells. Cell 161:1187-1201.

Kormelink TG, Arkesteijn GJA, Nauwelaers FA, van den Engh G, Nolte-'t Hoen EN, Wauben MH (2016) Prerequisites for the analysis and sorting of extracellular vesicle subpopulations by highresolution flow cytometry. Cytometry A 89:135-147.

Krishnaswami SR, Grindberg RV, Novotny M, Venepally P, Lacar B, Bhutani K, Linker SB, Pham S, Erwin JA, Miller JA, Hodge R, McCarthy JK, Kelder M, McCorrison J, Aevermann BD, Fuertes FD, Scheuermann RH, Lee J, Lein ES, Schork N, et al. (2016) Using single nuclei for RNA-seq to capture the transcriptome of postmortem neurons. Nat Protoc 11:499-524.
Krutzik PO, Nolan GP (2006) Fluorescent cell barcoding in flow cytometry allows high-throughput drug screening and signaling profiling. Nat Methods 3:361-368.

Krutzik PO, Clutter MR, Trejo A, Nolan GP (2011) Fluorescent cell barcoding for multiplex flow cytometry. Curr Protoc Cytom Chapter 6:Unit 6.31.

Lannigan J, Nolan JP, Zucker R (2016) Measurement of extracellular vesicles and other submicron size particles by flow cytometry. Cytometry A 89:109-110.

Libregts SFWM, Arkesteijn GJA, Németh A, Nolte-'t Hoen ENM, Wauben MHM (2018) Flow cytometric analysis of extracellular vesicle subsets in plasma: impact of swarm by particles of noninterest. J Thromb Haemost 16:1423-1436.

Luquet E, Biesemann C, Munier A, Herzog E (2017) Purification of synaptosome populations using fluorescence-activated synaptosome sorting. Methods Mol Biol 1538:121-134.

Macosko EZ, Basu A, Satija R, Nemesh J, Shekhar K, Goldman M, Tirosh I, Bialas AR, Kamitaki N, Martersteck EM, Trombetta JJ, Weitz DA, Sanes JR, Shalek AK, Regev A, McCarroll SA (2015) Highly parallel genome-wide expression profiling of individual cells using nanoliter droplets. Cell 161:1202-1214.

Nolan JP (2015) Flow cytometry of extracellular vesicles: potential, pitfalls, and prospects. Curr Protoc Cytom 73:13.14.1-13.14.16.

Nolan JP, Stoner SA (2013) A trigger channel threshold artifact in nanoparticle analysis. Cytometry A 83:301-305.

van der Pol E, van Gemert MJC, Sturk A, Nieuwland R, van Leeuwen TG (2012) Single vs. swarm detection of microparticles and exosomes by flow cytometry. J Thromb Haemost 10:919-930.

van der Pol E, Coumans F, Varga Z, Krumrey M, Nieuwland R (2013) Innovation in detection of microparticles and exosomes. J Thromb Haemost 11 [Suppl 1]:36-45.

Postupna NO, Keene CD, Latimer C, Sherfield EE, Van Gelder RD, Ojemann JG, Montine TJ, Darvas M (2014) Flow cytometry analysis of synaptosomes from post-mortem human brain reveals changes specific to Lewy body and Alzheimer's disease. Lab Invest 94:1161-1172.

Poulopoulos A, Murphy AJ, Ozkan A, Davis P, Hatch J, Kirchner R, Macklis JD (2019) Subcellular transcriptomes and proteomes of developing axon projections in the cerebral cortex. Nature 565: 356-360.

Prieto GA, Trieu BH, Dang CT, Bilousova T, Gylys KH, Berchtold NC, Lynch G, Cotman CW (2017) Pharmacological rescue of long-term potentiation in Alzheimer diseased synapses. J Neurosci 37:11971212.

Robinson PJ, Gehlert DR, Sanna E, Hanbauer I (1989) Two fractions enriched for striatal synaptosomes isolated by Percoll gradient centrifugation: synaptosome morphology, dopamine and serotonin receptor distribution, and adenylate cyclase activity. Neurochem Int 15:339-348.

Simonsen JB (2016) A liposome-based size calibration method for measuring microvesicles by flow cytometry. J Thromb Haemost 14:186-190.

Stoner SA, Duggan E, Condello D, Guerrero A, Turk JR, Narayanan PK, Nolan JP (2016) High sensitivity flow cytometry of membrane vesicles. Cytometry A 89:196-206.

Taupin P, Zini S, Cesselin F, Ben-Ari Y, Roisin MP (1994) Subcellular fractionation on Percoll gradient of mossy fiber synaptosomes: morphological and biochemical characterization in control and degranulated rat hippocampus. J Neurochem 62: 1586-1595.

Terrian DM, Johnston D, Claiborne BJ, Ansah-Yiadom R, Strittmatter WJ, Rea MA (1988) Glutamate and dynorphin release from a subcellular fraction enriched in hippocampal mossy fiber synaptosomes. Brain Res Bull 21:343-351.

Vaaga CE, Borisovska M, Westbrook GL (2014) Dual-transmitter neurons: functional implications of co-release and cotransmission. Curr Opin Neurobiol 29:25-32. 
Vida TA, Emr SD (1995) A new vital stain for visualizing vacuolar membrane dynamics and endocytosis in yeast. J Cell Biol 128: 779-792.

Wersto RP, Chrest FJ, Leary JF, Morris C, Stetler-Stevenson M, Gabrielson E (2001) Doublet discrimination in DNA cell-cycle analysis. Cytometry 46:296-306.
Whittaker VP (1993) Thirty years of synaptosome research. J Neurocytol 22:735-742.

Williams C, Shai RM, Wu Y, Hsu Y-H, Sitzer T, Spann B, McCleary C, Mo Y, Miller CA (2009) Transcriptome analysis of synaptoneurosomes identifies neuroplasticity genes overexpressed in incipient Alzheimer's disease. PLoS One 4:e4936. 